\title{
A Hybrid Learning Approach for Modelling and Analyzing Clickstream Data from
}

\section{Learning Management Systems}

by

\section{Alexis Amezaga}

A thesis submitted to the Faculty of Graduate and Postdoctoral Affairs in partial fulfillment of the requirements for the degree of

Master of Information Technology

In

Digital Media with Specialization in Data Science

Carleton University

Ottawa, Ontario

(c) 2021, Alexis Amezaga 



\section{Abstract}

In this research, a hybrid approach combining a Hidden Markov Model (HMM) with a Long Short-Term Memory (LSTM) recurrent neural network (RNN) is introduced to model realtime online feedback to students when completing academic activities using online Learning Management Systems (LMS). The solution provided is a Smart Classifier which unravels, and processes hidden patterns in the data to train appropriate metrics to raise flags indicating outlier student behavior based on historical data from previous and ongoing sessions.

This work introduces an approach that facilitates modifications of the attention mechanism in Transformer models. Using this approach, the predictor module of the proposed solution is improved. The key element of this improvement is to use a Bayesian Graph Network (BGN) coupled to a Transformer. As a novelty, this method provides a systematic customization of the attention mechanism in Transformer models that can be applied to a range of problems involving clickstream data. 



\section{Acknowledgements}

Before anything else, I wish to express my deepest gratitude to my supervisor, Dr. M. Omair Shafiq for his unwavering support and belief in me. This thesis and the research behind it have been continuously guided by his enthusiasm, knowledge, research and teaching excellence in the field. From the early stages of my transition into the program and subsequent course work, to the completion of this thesis under the remote work and studying conditions imposed on us all by the pandemic, his remarkably constructive and effective feedback, insightful comments, and encouragement have been crucial for my success at every step of this journey. He has taught me very valuable lessons in a demanding field, always in the most professional, and yet warm and friendly, manner.

This work would not have been possible without the financial support of the Carleton University COVID-19 Rapid Response Research Grant, awarded to Dr. M. Omair Shafiq in 2020; and the funding opportunity from the School of Information Technology to undertake my studies. I would like to express my sincere thanks to Dr. Audrey Girouard, Associate Director, Graduate Studies for her overall guidance, and to the faculty and staff of the School of Information Technology for providing students with a stable and highly successful academic program amidst the unprecedented COVID-19 pandemic.

I would like to extend my special thanks to my professors in the program at the School of Information Technology and the School of Computer Science, especially Dr. Olga Baysal, Dr. Elio Velazquez, and PhD. Candidate Mohamed Abdelazez, for their valuable work in shaping some of the key methods and techniques applied in this research. I would like 
to express my gratitude to Dr. David Sprague for his kind help and support allowing me to contribute to his Teaching Assistance team, gaining valuable and practical knowledge while helping students through the last two years.

I would like to thank all members of the Administrative Staff at the School of Information Technology, and especially Ria Akaiwa and Erenia Hernandez Oliver, Graduate Administrators for their outstanding efforts to make my academic life much easier. I also extend my thanks to Andrew Pullin, Sr. Software Designer of the Openstack computer facility from the School of Computer Science for always kindly accomodating my needs for technical support when required. My appreciation also goes out to my colleagues in the program, and especially Leroy Ngene for his time devoted to sharing advice.

Finally, I would like to express my deepest thanks to my lovely wife Ana, for her daily support; to my sister, and my brother for their encouragement all through my journey in this program of studies. 


\section{Contents}

$\begin{array}{lll}\text { Abstract } & \text { i }\end{array}$

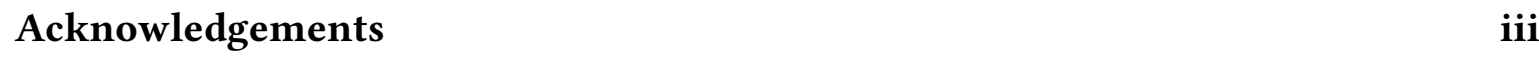

1 Introduction $\quad 1$

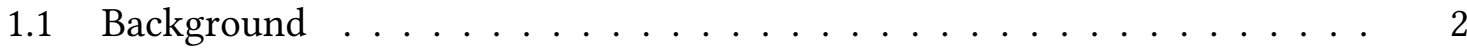

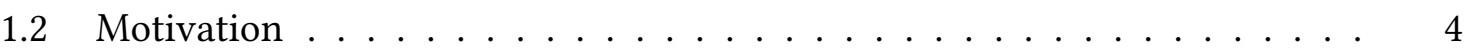

1.3 Thesis Objectives ........................... 5

1.4 Summary of the Thesis' Contributions . . . . . . . . . . . . 5

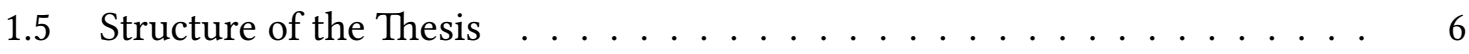

2 Literature Review and Related Work $\quad 8$

2.1 Pattern Detection in Sequential Data . . . . . . . . . . . 8

2.2 Machine Learning in Sequential Data Prediction . . . . . . . . . . . . 11

2.3 Learning Management System Overview . . . . . . . . . . . . . . . 18

3 Modeling and Predicting Online Activities of Students 21

3.1 Capturing User Behavior Patterns from Clicktream Data within the LMS . 21

3.2 The Smart Classifier . . . . . . . . . . . . . . . . . . . . . 24

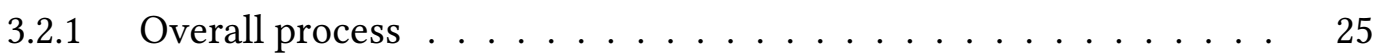




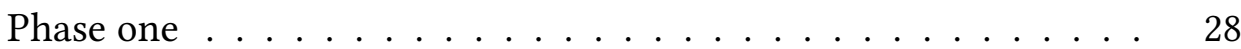

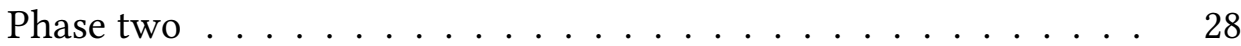

3.2 .2 Data description . . . . . . . . . . . . . . . . 29

3.2 .3 System model . . . . . . . . . . . . . . . . . . . . 31

The HMM component . . . . . . . . . . . . . . . 31

The LSTM component . . . . . . . . . . . . . . . . 33

The Classifier component . . . . . . . . . . . . 36

3.2.4 Model architecture . . . . . . . . . . . . . . . . . . 39

3.2.5 Algorithm design . . . . . . . . . . . . . . . . . 41

3.2.6 Design discussions . . . . . . . . . . . . . . . . . 45

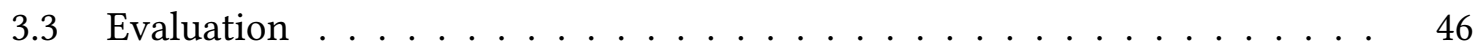

3.3.1 Complexity analysis ... . . . . . . . . . . 46

3.3.2 Efficiency analysis . . . . . . . . . . . . . . 46

3.3.3 Experiments ......................... 47

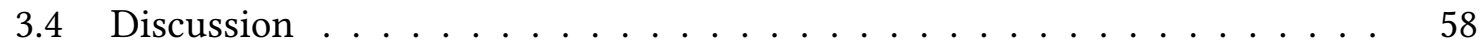

4 Transformer with Modified Attention Mechanism $\quad 61$

4.1 Predicting User Decision with Clikstream Data . . . . . . . . . . . . 61

4.2 The Modified Transformer . . . . . . . . . . . . . . . . 64

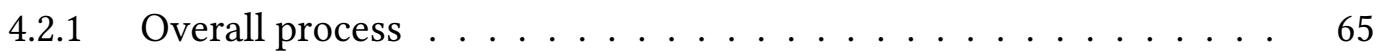

4.2 .2 Data description $\ldots \ldots \ldots \ldots \ldots$

4.2 .3 System model . . . . . . . . . . . . . . . . 67

The Bayesian Graph Network . . . . . . . . . . . . . 67

Encoder-Decoder Systems _. . . . . . . . . . . . . . . 69

Attention Mechanism _. . . . . . . . . . . . . . 69 
4.2 .4 Architecture . . . . . . . . . . . . . . . . 71

$4.2 .5 \quad$ Algorithm design . . . . . . . . . . . . . . . . . . . . 74

4.2.6 System implementation details . . . . . . . . . . . . . . . 75

Attention modification . . . . . . . . . . . 77

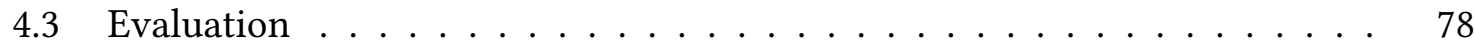

4.3.1 Complexity analysis . . . . . . . . . . . . . 79

4.3.2 Predictive performance . . . . . . . . . . . . . 79

4.3.3 Pattern detection performance . . . . . . . . . . . . . 83

Efficiency analysis: Time vs Performance tradeoff . . . . . . . . . 83

4.4 Discussion . . . . . . . . . . . . . . . . . . 84

5 Conclusions and Future Directions $\quad 86$

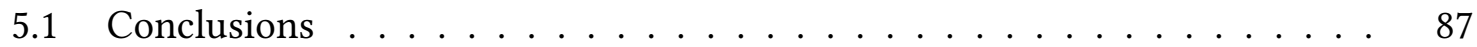

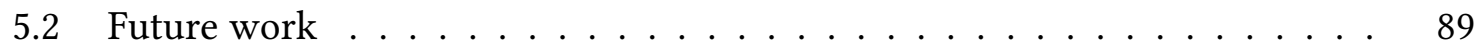

$\begin{array}{ll}\text { Bibliography } & 91\end{array}$ 


\section{List of Tables}

2.1 Comparative Analysis Table . . . . . . . . . . . . . . 15

3.1 Data Features Description _. . . . . . . . . . . . . . 29

3.2 Score Combinations for Flag Activation . . . . . . . . . . . . . . . 44

3.3 Adjusting LSTM Memory Window . . . . . . . . . . . . . . . . . . 49

3.4 Predictor Module Performance . . . . . . . . . . . . . . . . . . . . . . . 49

3.5 Thresholds Scores for Pattern Detection . . . . . . . . . . . . . 51

3.6 One-Signal Configuration . . . . . . . . . . . . . . . . 54

3.7 Multi-Signal Configuration . . . . . . . . . . . . . . 55

3.8 Smart-Classifier Usability . . . . . . . . . . . . . . . . 57

4.1.a BGN Configuration for Transformer (GRU) . . . . . . . . . . . . . . . 80

4.1.b BGN Configuration for Transformer (LSTM) . . . . . . . . . . . 81

4.2 Predictive Capability of Model's Architectures . . . . . . . . . . . . 82 


\section{List of Figures}

1.1 The Pattern Detection Problem . . . . . . . . . . . . . . . . . . . 3

3.1 Solution Workflow . . . . . . . . . . . . . . . . . 25

3.2 High-Level System Diagram . . . . . . . . . . . . . . . . . 27

3.3 Clicstream Data Sample . . . . . . . . . . . . . . . . . 30

3.4 Internal Mechanism of the LSTM Unit Cell . . . . . . . . . . . . . . . . . 34

3.5 Detail Technical Design . . . . . . . . . . . . . . . . . . . 40

3.6 Model Flow Chart . . . . . . . . . . . . . . . . . . . . . . 42

3.7 Example of the Outlier Detection Task . . . . . . . . . . . . . . . 45

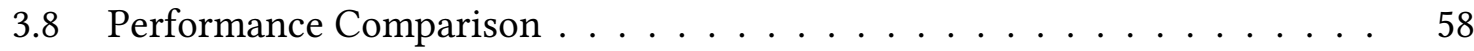

4.1 GRU Cell Unit . . . . . . . . . . . . . . . . . . . . . . . . . . . . 70

4.2 Predictor Component Flow Chart . . . . . . . . . . . . . 72

4.3 Bayesian Graph Network . . . . . . . . . . . . . . . . . . 73

4.4 Clickstream Scoring Process . . . . . . . . . . . . . . 75

4.5 Sequence-to-Sequence Architecture . . . . . . . . . . . . 76

4.6 Modified Transformer Architecture . . . . . . . . . . . . . . . . 77

4.7 Tradeoff: Time vs Performance . . . . . . . . . . . . . . . . . . . 83 


\section{List of Abbreviations}

$\begin{array}{ll}\text { BGN } & \text { Bayesian Graph Network } \\ \text { LMS } & \text { Learning Management System } \\ \text { LSTM } & \text { Long Short Term Memory } \\ \text { RNN } & \text { Recurrent Neural Network } \\ \text { Deep-RNN } & \text { Deep Recurrent Neural Network } \\ \text { DNN } & \text { Deep Neural Network } \\ \text { HMM } & \text { Hidden Markov Model } \\ \text { MC } & \text { Markov Chain } \\ \text { GRU } & \text { Gated Recurrent Unit } \\ \text { ML } & \text { Machine Learning } \\ \text { ANN } & \text { Artificial Neural Network } \\ \text { CNN } & \text { Convolutional Neural Network } \\ \text { SVM } & \text { Support Vector Machine } \\ \text { AI } & \text { Artificial Intelligence } \\ \text { ARIMA } & \text { Auto Regressive Integrated Moving Average } \\ \text { GA } & \text { Genetic Algorithm } \\ \text { TSC } & \text { Time Series Classification } \\ \text { CE } & \text { Natural Language Processing } \\ \text { NPL } & \end{array}$


To my lovely wife ... 



\section{Chapter 1}

\section{Introduction}

This chapter presents an overall description of the research work behind this thesis. Overall, the main goal of the work is to improve the interpretability of results when modelling clickstream data from online activity to gain insights in predicting human decisions, while keeping the model within a reasonable range of implementation complexity.

This thesis integrates two research phases. The first phase consists in the development and adjustment of an algorithm to timely detect user's behaviour patterns from Clickstream data while interacting with a Learning Management System (LMS). Clickstream data refers to a detailed log of how participants navigate through an online platform during a working session. The log typically includes the pages that the students visit to work on a particular activity, the time that they spend on each page, how they arrive at the page, and which page they could visit next, as well as, keystrokes and mouse/trackpad clicks and movements.. The focal point of this algorithm is to extract meaningful knowledge from the detected patterns that, in turn, could be returned to the user (i.e. the clickstream data generator) in the form of useful feedback in quasi-real time. 
The second phase of the research is concerned with improving the predictor module of the Smart Classifier that is developed in the first phase of the research. Improving the predictor module relies on a method developed here to modify the attention mechanism of a Transformer model used for the forecasting engine.

The main contribution of the second phase is the use of a Bayesian Graph Network (BGN) model combined with a Transformer model. This facilitates to customize the attention mechanism, and to obtain better scores for both, the predictive task and the overall performance of the Smart Classifier.

\subsection{Background}

Researchers have focused on the advantages of Machine Learning (ML) models to succeed regardless of the size of data (Chen et al., 2018); a survey of forecasting techniques to predict stock price is assessed. Some of the techniques included in the study are time series analysis, Artificial Neural Network (ANN), Convoluted Neural Network (CNN), Support Vector Machine (SVM), Long Short-Term Memory-NN (LSTM), and others (Fawaz et al., 2019). An enormous amount of research effort has been dedicated to the design of efficient outlier detection techniques, while taking into consideration efficiency, accuracy, high-dimensional data, and distributed environments, among other factors (Wu and Chan, 2020). In fact, the classification of dynamical data streams is among the most complex tasks in ML classification.

The chart in Figure 1.1 illustrates the problem of pattern detection that is shared by a variety 
of sequential data problems. When the pattern detection task is used to make inferences about human behaviour, or more precisely, human decision making, the need for methods that facilitate the interpretability of the model output becomes crucial, adding complexity to the model.

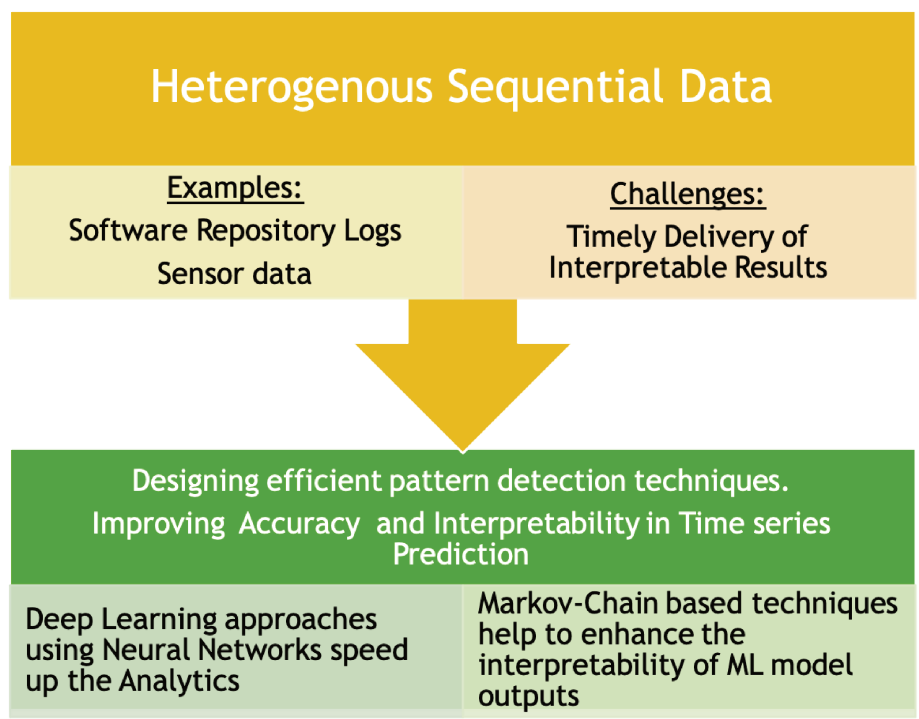

Figure 1.1: The Pattern Detection Problem

To address the problem of detecting behavior patterns from interaction user-LMS's clickstream data, a methodology is introduced to combine a probabilistic inference model with a deep-learning approach, with the goal of facilitating the interpretability of the model output. To cover both interpretability and speed in the pattern detection task, this research focuses in the designing of a complementary system (or plug-in) for LMS that processes data from interactions between students and LMS in quasi-real time as they carry out dayto-day education and learning activities. Essentially, a hybrid Artificial Intelligence (AI) model is proposed, which combines deep-learning techniques with probabilistic methods. A Deep Recurrent Neural Network (Deep-RNN) is merged with Markov-Chain (MC) based 
models, such as a Hidden Markov Model (HMM) and a Bayesian Graph Network (BGN). The result of this merge is a Smart Classifier, which detects hidden patterns in the data coming from the interactions of students with LMS. A key challenge addressed by this work is the extremely difficult task of predicting people's behaviour. The predicting task is complicated by the need for a quasi-real time response, which often implies limited and somewhat erratic datasets within an online session.

\subsection{Motivation}

The motivation for this work is, primarily, a contribution to provide better support to the students in their interactions with LMS.

All computer applications generate a trace log data that allows to properly address any problem that could be causing the system not to work as expected. This is specially the case for the data generated by the user in their interactions with the computer applications. In the case where the interaction between User \& System becomes the interaction between Student \& LMS, the log data from this interaction offers a rich source from which meaningful knowledge can be extracted and delivered directly to the students in the form of useful support.

In the context of the global pandemic, online learning platforms have become more important than ever, adding to the need for the AI-powered tools that are developed in this work. Given that all benefits in the use of LMS are interconnected, the goal is to enhance the overall learning experience for the students, while keeping them engaged. The approach 
in this work contributes novel features, while expanding on recent research.

\subsection{Thesis Objectives}

In this work, research goals have been fuelled by the desire of developing a model with the ability to capture relevant information in data from the interaction between a user and an LMS with the potential of using that knowledge in helping to create a tool that could eventually be used to provide meaningful feedback to the user in quasi-real time. The need to create a model that is able to deliver a response in quasi-real time is justified by the potential uses of such model which could include the implementation of a system to provide assistance to a user interacting with an LMS in real-time. In that context, the questions to be answered can be summarized as:

RQ1: how to detect both regular and outlier patterns in clickstream data from an LMS?, and how the appearance of outlier patterns in the data could be anticipated, so that the detection task occurs in quasi-real time?, and

RQ2: would it be possible to introduce a measurable score that could captures some hidden dynamics in the data-generation process that could be used to enhance the overall performance of the pattern-detection task?

\subsection{Summary of the Thesis' Contributions}

Driven by the questions presented, the research output of this work can be highlighted in the following main contributions: 
- The development of an algorithm to timely capture patterns from clickstream data by combining Markov Chain methods with recurrent neural networks for both classification and predictive tasks; and

- The introduction of a methodology to customize the attention mechanism in a Transformer model using probabilistic inferential methods in a manner that incorporates knowledge extracted from non-homogeneuos clickstream data type, e.g. including both categorical and numerical data. In this case, a BGN model was used as the probabilistic approach to model the clickstream data flow. The methodology proposed could potentially be applied beyond the scope of the LMS study-case presented in this thesis.

The solution presented is a model to process and classify patterns in multivariate time series with non-homogeneous data. This solution has the potential to be used as a base for the development of a tool for an LMS. Another potential use of the hybrid model presented in this work could be to process multivariate time-series generated by multi-sensor systems recording non-homogeneous data.

\subsection{Structure of the Thesis}

In Chapter 2, a literature review is presented to discuss relevant work around the two main problems of this thesis, (a) outlier pattern detection in sequential data, and (b) interpretability in sequential data prediction. The problem of finding patterns on data that could reveal user behaviour when interacting with an LMS is framed in Chapter 3; then, the design and implementation of a prototype for the proposed Smart Classifier is described in detail, experiments and results of this phase are also presented. All details of the second stage of 
the research are the subject of Chapter 4, the design and implementation of the algorithm to modify the attention mechanism for the Transformer model and the experiment results from the integration of the upgraded predictor component of the Smart Classifier. Finally, general conclusions, limitations, and paths to future work complete the thesis in Chapter 5. 


\section{Chapter 2}

\section{Literature Review and Related Work}

In this chapter, a collection of related work is reviewed. Findings indicate that the path explored in this research helps with the treatment of non-homogeneuos sequential data, that is common in pattern detection problems when interpreting the ML modelling of sequential data. Interpretability enhancement is key in improving the usability of ML models.

\subsection{Pattern Detection in Sequential Data}

Hidden Markov-Chain techniques are often applied to solve integration and optimization problems in large dimensional spaces (Chang, 2019). In (Boukerche, Zheng, and Alfandi, 2020) the authors explore research on outlier detection by completing a thorough and stateof-the-art review. Their survey considers classic and new trends of the proximity-based class, but their focus is on the new methods, favoring projection-based work and small amount of user feedback. Instead of using more traditional methods, extracting representative features from complex and high-dimensional data could yield more precise results when using deep neural networks for outlier detection, instead. A disadvantage of deeplearning approaches is being sensitive to hyper-parameters for optimal performance, which 
could lead to time-consuming and difficult problems. Overfitting has been identified a second issue with deep neural networks and this requires large amounts of data, which are not always available.

A basic technique called Outlier Exposure is presented in (Hendrycks, Mazeika, and Dietterich, 2018). This is claimed to represent an improvement from current outlier detectors. The method detects new, anomalous, out-of-distribution cases by training a network heuristic with out-of-distribution samples. The authors find that, even for large-scale image tasks, their method finds good application in vision and natural language processing. Their method shows three main advantages over previous techniques, which are improved model calibration, allocation of more plausible densities to out-of-distribution samples, and low overhead due to its computational efficiency. Methods that detect anomalies frequently focus on the session. In (Du et al., 2017), however, the authors work with the log entries and develop a system that changes to introduce new patterns. Using Long Short-Term Memory (LSTM), they present DeepLog, a model that works with a system log to show natural language sequence. Their system processes log patterns, detecting anomalies, and learning from history, including timestamp, log key and parameter values. The method introduces online training. The method combines LSTM with density clustering for higher effectiveness.

Online classification for dynamic data streams is presented in (Hammer, Yazidi, and Oommen, 2018). The authors use the Bayesian and the new anti-Bayesian classification methods. Estimating the time-varying quantiles of the distributions for the classes is at the core if the anti-Bayesian process. In (Wu and Chan, 2020), Wu and Chan focus on detecting temporal 
patterns from multivariate time series in different locations. Their information-theoretic method identifies spatio-temporal patterns using a test on statistical significance. Associations among patterns that occur sequentially with changing time delays in single and multiple time series across spaces are identified in their work. One advantage of their approach is the applications for clustering and classification. Taha and Hadi collect and analyze a large collection of papers in (Taha and Hadi, 2019) that propose methods for detection of anomalies; the authors group the work in 12 classes of methods according to the anomaly definition. In this critical comparison, the number of parameters and the time complexity are highlighted as key issues in this field of research. Method performance is affected by the choice of parameters and big data applications are impacted by time complexity.

The machine-learning research work has developed methods to address the principal learning tasks. In (Wei et al., 2019), Dietterich formally describes these methods as sliding windows, recurrent sliding windows, hidden Markov models (HMM), conditional random fields, and graph transformer networks. The paper also formally focuses on the learning tasks. The authors of (Zhou et al., 2018) present a method called QuickStop, which they claim to be the fastest misinformation detection tool. The problem is proposed as an optimal stopping problem with asymmetric cost function with regards to misinformation. The solution suggested by this work is a threshold-based stopping rule based on the martingale theory, after proving that the problem is a Markov optimal stopping problem. Modelling memory effects in discrete-state random walks is presented by Chang in (Chang, 2019). The predictive accuracy of the model is estimated with cross-validation and information criteria, with the use of higher-order Markov chains. Outside of fixed-order multistep models, model searching is not explored. It is claimed that models could be fit by order sequentially 
assuming that search is not challenging for fixed-order models. The authors of (Zhou et al., 2018) assess a Markov chain predictive model with field data collected from Zhongshan City bike sharing system. The work projects the daily production and attraction of stations.

Despite recent outstanding improvements, pattern detection in sequential data remains a very active research area. Most advances are concentrated on sensor-data processing, but interesting proposals also address the pattern-detection problem from user-generated data, specially clickstream data which is the focus of this research. In this context, the use of hybrid approaches has been proved to be a good strategy.

\subsection{Machine Learning in Sequential Data Prediction}

In this context, ML models can also be applied to predict data sequences. Machine Learning (ML) algorithms have been applied to time series forecasting in different scenarios like forecasting power consumption, transportation and traffic forecasting, exchange rate movements, or commodity purchases. Techniques have grown in complexity and variety, from traditional Auto-Regressive Integrated Moving Average (ARIMA), or linear regressions, to several clustering techniques, or a variety of unsupervised learning algorithms. In view of the technical complexity, there is a need for model performance evaluation (Yin et al., 2019).

Researchers have focused on the advantages of ML models to succeed regardless of the size of data (Matsubara and Sakurai, 2019), or on the combinations of ML models that capture across-multiple-series features with individual-series approaches (Deshpande and Sarawagi, 2019). Techniques for time series selection have also grown in complexity with 
decision trees, including geo-localization, (Chatzigeorgakidis et al., 2019). The main goal of forecasting is to explain behavior and interpret data-driven trends instead of arriving at individual future values. In this area, ML models have also developed, from older applications of Genetic Algorithm (GA) (Kumar et al., 2011) to more recent approaches where the use of machine learning techniques such as deep neural networks (DNN) (Zhong and Enke, 2019).

High forecasting accuracy and good generalization are claimed as experimental improvements. In (Hanif et al., 2017), the authors use a hidden Markov model to compute algorithms and to model the series, by applying transition and emission probabilities. The challenges of hidden Markov models are listed such as learning about the model, the evaluation process and the parameter estimation. Viterbi, Bauch-Welch and forward-backward algorithms are presented as solutions to the issues listed. The state space model is also analyzed.

Non-stationary data is transformed into time series in (Jamshed, Mallick, and Kumar, 2020) with the use of discrete wavelet analysis. To analyze customer behavior and purchasing patterns over time, a reformed hybrid combination of convolutional neural network (CNN) with long short-term memory (LSTM) is developed. To obtain the concerned item set (frequent) at the end of the pattern, the authors use CNN. For finding the time interval among each pair of successive item sets, they apply LSTM. A progressive database that removes the obsolete data is used for mining the sequential pattern. To demonstrate robustness, the accuracy of this work is analyzed by examining traditional algorithms. An empirical study is showcased in (Fawaz et al., 2019) with the most recent DNN architectures for time series classification (TSC), including an analysis of model performance. Under a unified 
taxonomy of DNNs for TSC, the most effective deep learning applications in different time series domains are presented. Each approach is evaluated on a univariate TSC benchmark (the UCR/UEA archive) and 12 multivariate time series datasets. This is done by offering an open source deep learning framework that also applies each method.

The authors of (Liu, Song, and Huang, 2020) develop a real-time prediction correction method applied to transportation. The prediction correction is based on a DNN approach by reconstruction error. It is claimed that the parameter learned could be better analyzed by the use of the reconstruction error. Furthermore, this work uses the reconstruction error to directly modify the weight in the DNN. In (Du, Liu, and $\mathrm{Hu}, 2019)$ the authors categorize and review a comprehensive set of techniques for interpretable machine learning. Their goal is to contribute to a better understanding of strengths and limitations of the various interpretation frameworks. Key issues remain to be solved, despite the fast advance of interpretable machine learning methods. The authors in (Begleiter, El-Yaniv, and Yona, 2004) use variable order Markov models to develop algorithms for prediction of discrete sequences over a finite alphabet. Different prominent prediction algorithms are presented, including Context Tree Weighting (CTW), Prediction by Partial Match (PPM) and Probabilistic Suffix Trees (PSTs).

Prediction quality, as measured by the average log loss, is selected for an assessment of the methods. By looking at a number of large protein classification tasks, algorithms are also evaluated based on the predictors. The authors claim that a "decomposed" CTW (a variant of the CTW algorithm) and PPM show better results with sequence prediction. Predicting 
search times is the goal in (Tran et al., 2017), with the authors presenting how Markov models derived from search logs can be used as a method for evaluating predictions. Parameter estimation methods are developed for customizing the predictions based upon a few user events observed. Results are satisfactory regardless of simplicity of models. More challenging models could be considered, such as ranking positions of snippets, or the number of query reformulations, which is suggested for future work.

In a recent work, (Karadayı, Aydin, and Öğrenci, 2020) a hybrid deep autoencoder framework is presented, which uses the autoencoder scheme for anomaly detection. The model has a multi-channel convolutional neural network $(\mathrm{CNN})$ used as encoder, and an LSTM network as the decoder. One of the benefits of the styled proposed is that the framework can be easily implemented on data with mixed-type attributes. In addition, it scales well to multi- variate datasets with high number of features, which is an advantage over proximitybased methods. Markov chain approaches continues as a very robust approach. Mizera, Pang and Yuang examine a two-state Markov chain approach; they show that splitting the state space into two disjoint sub-spaces and approximating steady-state probabilities help to avoid the problem of generating biased results when biological systems are modelled using large probabilistic boolean networks (PBNs) (Mizera, Pang, and Yuan, 2018). In the context of natural language processing, (Yang et al., 2016) propose the Hierarchical Attention Network (HAN) that captures two basic insights about document structure. Instead of filtering for (sequences of) tokens, taken out of context, they apply context to uncover when a sequence of tokens is relevant. Recently, (Wu et al., 2020) develops a Transformerbased approach to forecasting multivariate time series data; as a novelty, they take advantage of self-attention mechanisms, so that they can capture complex dependencies of 
TABLE 2.1: Comparative Analysis Table

\begin{tabular}{l|l|l|l}
\hline \hline Data type & Data size & Approach & Performance \\
\hline Sensor data $^{1}$ & over 2.5 M of records & SVM (clustering) & Accuracy: 0.98 (ground-truth) \\
\hline NL sequences $^{2}$ & over 12 M of records & LSTM-RNNs & $\begin{array}{l}\text { Precision: 0.16 (with no data up- } \\
\text { date), 0.90 (with active data up- } \\
\text { date) }\end{array}$ \\
\hline Click-stream data $^{3}$ & Over 2.3 M of records & HMM (baseline) M3PP & $\begin{array}{l}\text { Acc. (HMM): 0.651 Acc. (M3PP): } \\
0.728\end{array}$ \\
\hline \hline
\end{tabular}

various lengths from time series data. Wu et. al. (Wu et al., 2020) also suggest that their model can be generalized to discover hidden relationship between two arbitrary points in spatio-temporal space. Huynh et. al. (Huynh et al., 2020) use an innovative shortcut to analyze sequential pattern lattices which evades reiterative data copies during execution.

Recently, in (Hatt and Feuerriegel, 2020), a novel Markov modulated marked point process (M3PP) model is developed with the goal of detecting users at risk of exiting with no purchase from clickstream data. Their M3PP approach covers both the sequence of pages visited and the temporal dynamics between them, i.e., the time spent on pages, which examines click-stream data in a comprehensive manner. A continuous time-marked-point process is developed. A continuous- time Markov process modulates the marked-point process to account for different latent shopping phases. A risk assessment analysis is also presented as a secondary contribution.

Table 2.1 above shows performance reported from two papers that can serve as a reference for the preliminary results from the approach presented in this work. Considering (Du et al., 2017), the approach to assess the "accuracy" for the solution proposed in this work, is 
equivalent to their Precision with no data update. In both cases, they have a ground-truth to be used to assess the model. In the (Matsubara and Sakurai, 2019) and (Hatt and Feuerriegel, 2020) cases, there were also known ground-truth to train the data. By contrast, in this work there is no enough data to build the ground-truth to drive the experiments, data from early stages of the running system is used to build the initial "ground truth". Ground-truth is building itself as metric thresholds for classification are determined by using previous entries for the current session, and later on, by updating these thresholds using subsequent sessions and when the data progressively increases.

Ding and collaborators in (Ding et al., 2019) are concerned with modeling time series prediction with the use of deep learning. The paper shows a new form of loss called Extreme Value Loss (EVL) for identifying future occurrences of extreme events. The models used are the LSTM and Gated Recurrent Unit (GRU) with Cross Entropy (CE). The efficacy of the approach is validated with experiments on synthetic data and two real datasets of stock and climate. The approach, however, is not suitable for multiple correlated time series.

Eichmann and collaborators, in (Eichmann et al., 2019), introduce Metro-Viz, which is a visual tool that could help data scientists in performing a method of data visualization with the aim of identifying anomalies in time series data. This could allow for results to be analyzed in deeper detail when anomalies are detected. Metro-Viz has interactive features (e.g., comparative analysis, what- if testing) supported by data management strategies. The article shows the use of this tool in multiple time series datasets and with various anomaly detectors. It is not clear, however, how this work could be applied to multidimensional time series analysis. 
Tian et. al., in (Tian et al., 2019), use the learned hidden state as a bridge to present a forecast with an interpretable re-constructor (IPR). This combines the sequence to sequence (seq2seq) model based on long short-term memory (LSTM) with interpretable data reconstruction. The authors claim that their IPR or re-constructor can both: a) regularize data based on the learned frequency, as well as, b) extract the time series components. Based on their experiments, the authors report their IPR method to show superiority over stateof-the-art and traditional methods, as measured with the MSE and the Theil's U-statistics (U). As a limitation of their report, their method is first named as an acronym in Figure 1 without having previously explained it. Their method shows inconsistent results with increasing forecast horizons and across datasets.

Cirstea and collaborators combine two models in (Cirstea et al., 2018). Convolutional neural networks (CNNs) and recurrent neural networks (RNNs) are presented with special emphasis on the accuracy of predictions for correlated time series. A CNN is used on each individual time series, the convoluted features are combined, and then an RNN is applied on top of the convoluted features.

Finally, Özen et. al., in (Özen, Atalay, and Yazici, 2019), evaluate the forecasting performance of a number of state-of-the-art machine learning models, with the use of meteorological time series data for temperature projections. Among the models tested are Random Forest, Convolutional Neural Network, Long Short-Term Memory and hybrid Convolutional Neural Network Long Short-Term Memory. Models used for the reference case are the Vector Auto-regression model, as a linear model, and the Multi-layer Perceptron, being 
a simple neural network; the RMSE is the performance metric tested. Accuracy is reported to be improved with the use of 10 -fold cross validation. With 10 static groups, experiments are repeated with various data groups in tests. The authors augment the number of folds to improve accuracy. Random forest is documented to perform well with small amounts of data, with no seasonality or trend. The deep neural network method is presented as problematic when revealing variable relevance, due to insufficient amount of data in their case.

From this close-eye look over the related work literature review, the lessons can be summarized as follows. First, different versions of RNN seem to be preferred when addressing time series forecasting. Second, when considering multivariate correlated sequential data, hybrid approaches are often adopted. These methods generally use some type of hybrid model to select the sequential data features that are likely to be impacted by correlation in the data. This could be helpful when the data to be processed is non-homogeneuos. Finally, from this review it emerges that applying probabilistic inference models facilitates model output interpretability, while deep learning techniques deliver a more efficient search for better performance.

\subsection{Learning Management System Overview}

This work is concerned with pattern detection in quasi-real time. This requires an effective algorithm for a better understanding of contextual patterns in sequential data, and it is also helped by sequence prediction techniques that can capture relevant pattern in the data.

With COVID-19 lockdowns, online learning helps with physical distancing regulations and 
reduces workplace risk. Online learning could have benefits that go beyond physical distancing measures. Online lectures and lab sessions allow access to a large amount of material for many students.

LMS tracks progress, allows for course collaboration, and helps with content delivery among other benefits. An LMS can be briefly described as follows:

- There is an instructor leading several activities and students engaging in the learning activities.

- Terminals that a student uses to connect to the LMS could be different, it depends on the design of the specific learning platform.

- All activities that students and educators perform are generating data logs that are collected by the system.

- It is important to note that activities performed on an LMS could be either:

- Time-constrained activities performed in real time by the student; or

- Self-paced activities, (such as reviewing, downloading or uploading content).

With the rise in online activities for study and work, it is increasingly important to contribute to better online tools. In this context, ML models that detect patterns and predict data sequences can be used to improve the online experience of students.

In the context of prediction problems for people behavior, and regardless of the specific area, the panoramic in model performances is quite diverse. Borisov et. al. (Borisov et al., 2016) present a Click Model based on user behavior which relies on what they call "distributed 
representation"; essentially, they represent each document by its associated click patterns that are observed in a search engine and use a LSTM approach to predict user behavior. Some data analytics on LMS have been reported (Graf and Kinshuk, 2008), (Maratea, Petrosino, and Manzo, 2017), (Cantabella et al., 2019), (Filvà et al., 2019).

Most of the studies reviewed here focus on analyzing user behaviour and pattern detection from interactions with LMS in a post-processing fashion, and do not address lower performance of models at early stages of the data processing, when data is relatively limited. In the approach applied in this work, the use of outlier-detection metrics could improve the overall performance of the pattern detection task, by using transition probabilities computed through a Markov-Chain based model. The approach presented is primarily focused on quasi-real time analytics. The designed system is meant to be focused, mainly, on activities performed in real time by the student. To test this approach, a case study is designed to explore, implement and evaluate concrete and innovative ways to enhance students' learning experience when interacting with LMS. 


\section{Chapter 3}

\section{Modeling and Predicting Online}

\section{Activities of Students}

Interpretability of ML models of sequential data that was widely analyzed in the previous chapter is explored in a more concrete case here. The goal in this chapter is to present work that applies a hybrid model for real-time online feedback to students when completing academic activities using online learning management systems (LMS). An HMM-LSTM hybrid approach is proposed. The solution presented here is a Smart Classifier empowered by a hybrid system combining a Markov-Chain (MC) approach, specifically a Hidden Markov Model (HMM), with a Long Short-Term Memory (LSTM) neural network.

\subsection{Capturing User Behavior Patterns from Clicktream Data within the LMS}

In this research, the early detection of patterns in sequential data is addresed. The solution to be presented in this paper, is a smart classifier empowered by a hybrid system combining a Markov-Chain (MC) approach, specifically a Hidden Markov model (HMM), with a Long 
Short-Term Memory (LSTM) neural network. One particular interest is the possibilities of the Markov Chain model in the analysis of pattern detection in general and outlier detection specifically. This idea could be used to monitor and detect specific behaviors, as a sequence of activities, in students from their interactions with online learning management systems (LMS). One of the applications could be the design of early recommendations to students with an aim to induce timely adjustment of the sequence of actions, eventually leading to better outcomes for students.

The methodology is to combine the MC-based model with a deep-learning approach (a LSTM neural network) to obtain predictions of categorical values for the features that represent the target the records of student activities (target). This additional complementary information describes time spent on one activity, number of clicks, keystrokes, mouse movements, and others, closely linked to the type of activity.

Deep learning methods, such as RNNs, have been very useful in prediction for sequential data. In particular, LSTM for time series has been reported to be powerful in this domain ((Du et al., 2017), (Jamshed, Mallick, and Kumar, 2020), and (Begleiter, El-Yaniv, and Yona, 2004)). First, the MC-based model will predict a short sequence of immediate actions into the future. Second, this forecast sequence will have a set of additional information, that is number of clicks, keystrokes, etc., which will be projected with the use of the LSTM. Third, the model will evaluate the predicted sequence with its attached projected additional information and assign a label as "regular" or "non-regular" pattern. Non-regular pattern is also referred to as outlier in this work. Non-regular patterns are the subsequences that are classified as outliers, given its scores according to the outlier-detection metrics used in this 
work, which are miss probability, miss rate, and entropy reduction (Jha, Tan, and Maxion, 2001).

Using a different approach, each one of these metrics evaluates all transition probabilities between states in the analyzed subsequence and score it. The greater the score is, the smaller odds for this subsequence. When the score is higher than the fitted threshold for the metric, the subsequence is considered an outlier. In this context, the outlier subsequences are said to exhibit an outlier pattern. Finally, alerts or flags could be raised to warn students when necessary in the hopes that the student use the information to steer their actions into the right direction. The model evaluates the previous and predicted future behaviors, analyzes and raises flags before the predicted future behavior actually occurs.

In the specific case of an academic exercise, such as a lab, for which students are evaluated, a system with these characteristics could also be trained to identify the behaviour that potentially can lead to better student performaces.

In summary, the problem of interpreting the student online behaviour activity is to help them in optimizing and redirecting its activity for success, viewed from a data science perspective, which can be written as: Given a multivariate time-series featuring non-homogeneous data being dynamically generated by a user interacting with an online platform, how to detect patterns in the multivariate time series from real-time generated clickstream data, to activate a warning system in quasi-real time? The focus is to consider non-homogeneous data being generated by users interacting with an online platform, such as a Learning Management System. The research question driving this stage of the research is: 
RQ: How a hybrid approach, using Markov-Chain based methods and RRNs, could classify patterns and detect outliers in clickstream data by actively analyzing data of users interacting with an online platform?

\subsection{The Smart Classifier}

The proposed solution uses data that is produced by students interacting and performing activities in online LMS, e.g., viewing course material, taking quizzes, tutorial or lab sessions, and etc. The online activity data is produced as clickstream data and is well-structured.

The proposed solution is a Smart Classifier system that works as a hybrid deep-probabilisticoutlier-detector and that is powered by a hybrid model combining a Hidden Markov Model (HMM), and a Long-Short Term Memory (LSTM) neural network. The hybrid model includes a sequence predictor which feeds the classifier to raise alerts and flags as output of the system. The overall workflow of the process of the proposed system is represented in Figure 3.1. 


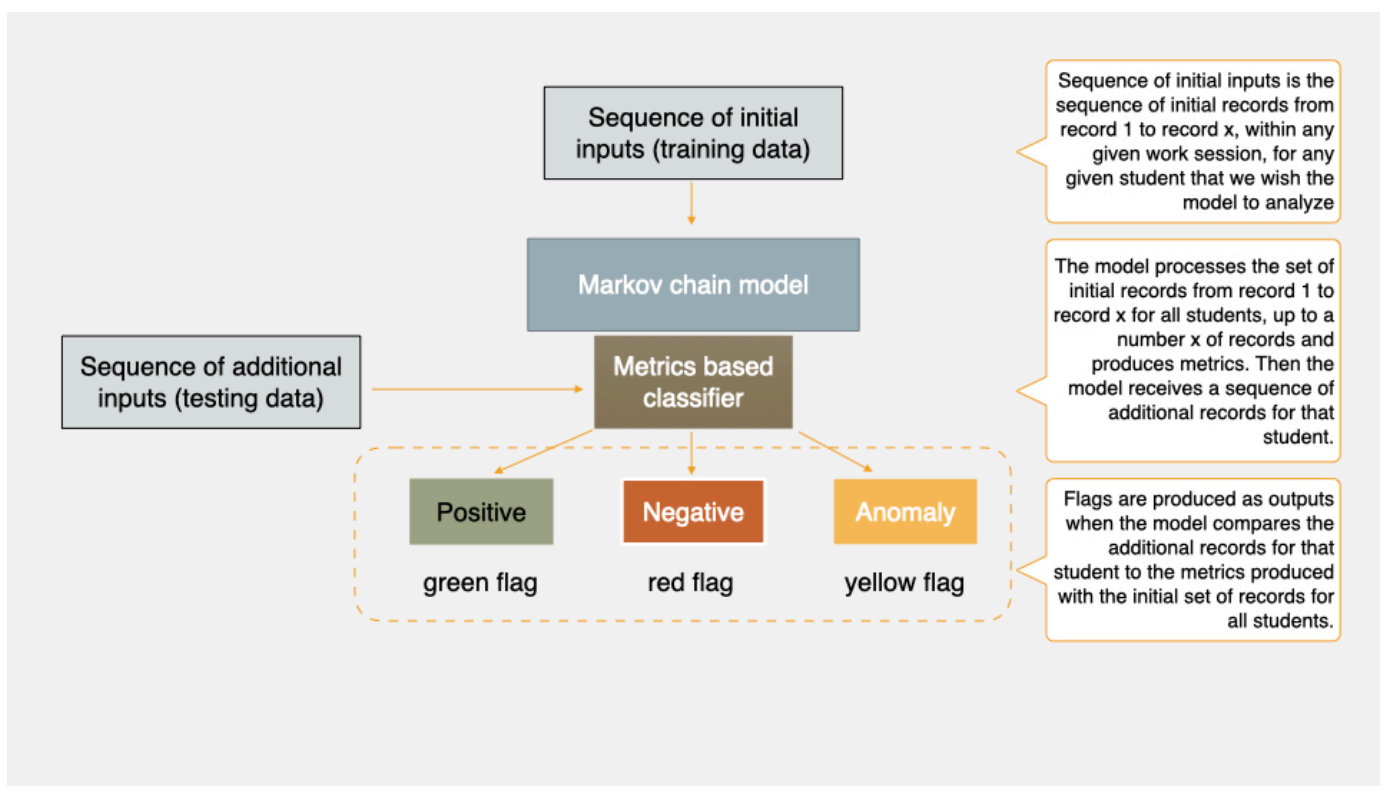

FIGURE 3.1: Solution Workflow

The use of deep learning to improve MC-based models in sequential data analysis is not new. This area of research has been reported in [1, 3](Matsubara and Sakurai, 2019), (Fawaz et al., 2019). The novelty in this work is the use of deep learning, in this case an LSTM model, along with Markov Chain, and preprocessing of data, for predictive analysis of time series associated to the sequence with the goal of improving classification. The metrics that inform the classifier rely on the output of the LSTM model. LSTM prediction results are used to guide the construction of the metrics-based classifier.

\subsubsection{Overall process}

The data sequences and additional information are gathered from a collecting system and processed dynamically by the hybrid smart classifier, which generates alert flags (i.e. positive, negative, or anomalies). The flags activate an alert assistant that provides an informed 
feedback to the student, or to an assistant who timely will provide meaningful tips and recommendations to the student. This process is sketched in Figure 3.2.

The system is fed with logs that represent activities as students interact with LMS. The incoming data is preprocessed and is structured in a tabular format, which is later stored as comma-separated-values (CSV) data (this preprocessing is not part of this work). The data preprocessing operates as an independent module, and its output is connected to the Smart Classifier.

After the stream of data generated by the students' activity logs is connected, the system continuously loads the data into a dataframe in real-time. The system first collects enough data depending upon the type of activities students perform in order to train a first version of the MC-HMM model, which is the initial probability vector, and transition matrix for the main variable and for additional variables, and start preparing the LSTM neural network that will be used.

The variables included in the multivariate time series of online activity for each student are, session number, current activity, initial timestamp for the current activity, time spent on current activity, number of clicks (left and right), keystrokes, and mouse movements (distance covered by the mouse). 


\section{High-Level System Diagram}

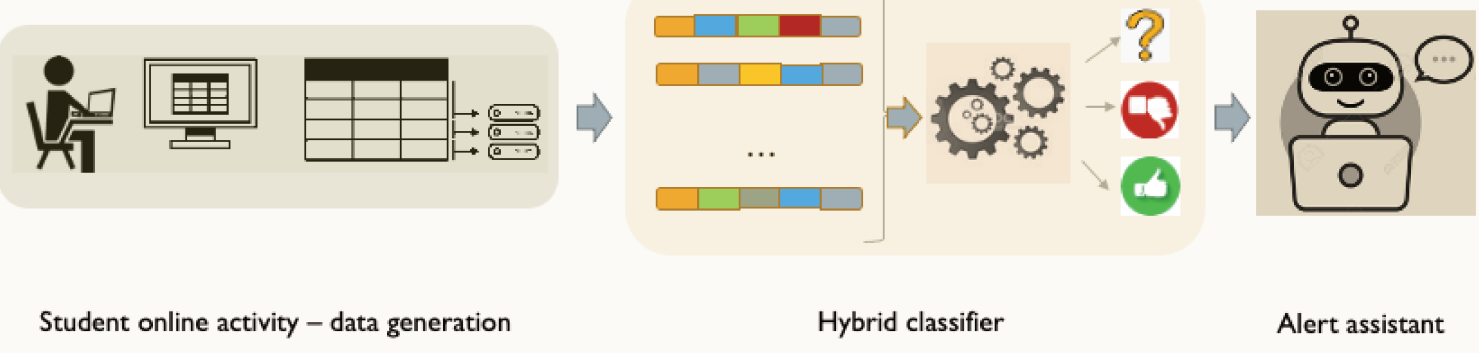

FiguRE 3.2: High-Level System Diagram

The hybrid classifier system, the Smart Classifier, proposed here has three components: (i) the HMM component, (ii) the LSTM component, and (iii) the pattern detector component (with previously optimized default-thresholds). From here on, the Classifier is defined as a combination of the HMM with the pattern detector. The system works as follows:

1. The data is loaded into the system and a 1st order $\mathrm{MC}(\mathrm{MC}-\mathrm{O}(1))$ model is built for the target variable, which is the activity the student is currently working on.

2. The HMM component of the system is built for the same target variable, considering a set of other variables as hidden variables.

3. Once the MC-O(1) is ready, the Classifier is trained with the data; and the LSTM component of the system is trained for the target variable.

4. Using the LSTM component, a sub-sequence is predicted and then assessed by the Classifier.

5. Finally, a flag (red, yellow, or green) is raised depending on the Classifier's output. The whole system will continue with its session-activity monitoring routine. 
In section 4.6 System implementation details, the above process is fully described. In the solution, when the system is building its HMM component (i.e. computing the initial probability vector and the transition matrix), simultaneously the LSTM component of the system will be training a predictor for the time spent in future activities inside the session. This implies that both the transition matrix calculation and the LSTM forecast should be executed in parallel. In short, the proposed solution will facilitate to offer timely suggestions to students interacting with an institutional platform, it will provide useful insight to Lab assistants, while at the same time, it will help to improve education supporting platforms.

\section{Phase one}

This is a description of the proposed solution in more detail. In an initial phase, the space state is determined for both the target variable of the model, Activity, and the variable used as auxiliary variable in the HMM. in this case, the spending time variable is used to build the HMM. Next, first-order transition matrices are computed for both variables and the emission matrix for the auxiliary variable. At this point, these Markov-based components are ready to train the Classifier component of the model. The first running cycle, the data for all students is processed to estimate the first set of threshold values for the outlier-detection metrics, and then the data for every student is processed individually, with outlier-detection metrics threshold being updated as the data increases.

\section{Phase two}

In a second phase, the LSTM is built. The LSTM will follow these steps:

- Proper encoding is applied to the target variable, which is Activity, first column in the sample table on Figure 3.3. 
- The features are engineered to prepare the LSTM inputs by using the data from the whole session until the current time.

- The LSTM model is initialized. Four hidden layers are used for the network each one of them followed by dropout layers.

- Once the LSTM model is trained for each student data in each session (about a hundred students per session)

\subsubsection{Data description}

Data from student online activity will be used to test the potential of this method for uncovering hidden relationships among selected data features that may indicate the probability of success in a particular set of online activity strategies. In Table 3.1, the description of features of the clickstream dataset used in this workbook is presented.

TAble 3.1: Data Features Description

\begin{tabular}{l|l}
\hline \hline Feature & \multicolumn{1}{c}{ Description } \\
\hline activity & activities are labeled based on the title of web pages that are on focus of the student. \\
\hline start_time & the start date and time of a specific activity with the format: dd.mm.yyyy hh:mm:ss \\
\hline end_time & the end date and time of a specific activity with the format: dd.mm.yyyy hh:mm:ss \\
\hline idle_time & duration of idle time between the start and end time of an activity in milliseconds. \\
\hline mWheel & amount of mouse wheel during an activity. \\
\hline mWheel_C & number of mouse wheel clicks during an activity. \\
\hline mClick_L & number of mouse left clicks during an activity. \\
\hline mClick_R & it shows the number of mouse right clicks during an activity. \\
\hline m_mov & distance covered by the mouse during an activity, expresed in number of pixels. \\
\hline KyS & number of keystrokes during an activity. \\
\hline \hline
\end{tabular}


Data on online activity from the interaction of 115 students with an LMS is collected (Vahdat et al., 2015). All users are engaged in six designed work sessions, $w_{1}$ to $w_{6}$, to complete one laboratory assignment and obtain one final grade, with the highest grades indicating success and the lower grade or absence of records indicating failure. Some students failed to participate in some of the work sessions, but in principle, each user would have had the opportunity to complete the six work sessions. For each student $s$, each work session $w$ produced a set of records, from $r_{1}$ to $r_{M}$. A selection of features included in the data is shown on Figure 3.3.

\begin{tabular}{|l|l|l|r|r|r|r|r|r|r|r|}
\hline Activity & start_time & end_time & idle_time & mWheel & mWheel_C & mClick_L & mClick_R & m_mov & Kys \\
\hline Other & $2.10 .201411: 25: 33$ & $2.10 .201411: 25: 34$ & 0 & 0 & 0 & 0 & 0 & 84 & 0 \\
\hline Aulaweb & $2.10 .201411: 25: 35$ & $2.10 .201411: 25: 42$ & 218 & 0 & 0 & 4 & 0 & 397 & 0 \\
\hline Blank & $2.10 .201411: 25: 43$ & $2.10 .201411: 25: 43$ & 0 & 0 & 0 & 0 & 0 & 59 & 0 \\
\hline Deeds & $2.10 .201411: 25: 44$ & $2.10 .201411: 26: 17$ & 154117 & 6 & 0 & 8 & 0 & 1581 & 4 \\
\hline Other & $2.10 .201411: 26: 18$ & $2.10 .201411: 26: 18$ & 0 & 0 & 0 & 2 & 0 & 103 & 0 \\
\hline Other & $2.10 .201411: 26: 19$ & $2.10 .201411: 26: 27$ & 460 & 0 & 0 & 4 & 0 & 424 & 8 \\
\hline Blank & $2.10 .201411: 26: 28$ & $2.10 .201411: 26: 28$ & 0 & 0 & 0 & 1 & 0 & 93 & 0 \\
\hline
\end{tabular}

FIgURE 3.3: Clicstream Data Sample

The focus of the analysis is on any given record $r$, which could also be defined as a sequence of activities and features. For each students $s$ and each work session $w$, each record $r$ is a matrix or set of time series with time $t_{1}$ to $t_{F}$. The time series in each students record $u_{i}$ are:

- the activity type, which has a string format and is one character identified with a letter, there are about nine activity types;

- the duration of the activity in seconds, which is a numeric format time series; and 
- about four additional time series that define up to four additional features.

\subsubsection{System model}

The approach selected for this work takes into consideration a number of successful hidden Markov-Chain models, and neural network models that have used LSTM networks as well.

\section{The HMM component}

As it has been explained above, a component of the model in this work is a Markov-Chain (MC) process (Hanif et al., 2017). In the MC process, the system is a sequence with $N$ elements or states. Each state belongs to the state space vector $Q$, the set of all possible states accessible to the system.

Let $Q=\left\{q_{1}, q_{2}, \cdots, q_{r}\right\}$ be the state space vector of the system. In this work, $Q$ is the set of all different activities a user could be working on during a session, this means, each $q_{i}$ denotes an activity available to the user.

The components of the initial probability vector are the frequencies of each component of the state space vector, $\vec{\Pi}=\left\{\pi_{1}, \pi_{2}, \cdots, \pi_{r}\right\}$, where $\pi_{i}=P\left(s_{0}=q_{i}\right)=\frac{1}{N} \times$ [total count of $\left.q_{i}\right]$.

The probabilities of $1^{\text {st }}$ order transition from one state to another are all set by the Markov Chain transition matrix, $\mathbf{A}=\left\{a_{i j}\right\}$, where $a_{i j}=P\left(s_{t}=q_{j} \mid s_{t-1}=q_{i}\right)$. This is, the probability that the system will go from a given state $q_{i}$ to another state $q_{j}$ is given by $a_{i j}$. 
The main feature of Markov Chain models is the it Markov Property, which states that the probability that one state will transition to another depends only upon the present state. This is as if the system has no memory of older states.

$$
P\left(s_{t}=q_{j} \mid s_{t-1}=q_{i}, s_{t-2}=q_{k}, \cdots\right)=P\left(s_{t}=q_{j} \mid s_{(t-1)}=q_{i}\right)
$$

An important consequence of the Markov Property is that the probability of a particular subsequence in the process is given by

$$
P\left(q_{i}, q_{j}, q_{k} \cdots, q_{v}\right)=\pi_{i} \cdot a_{i j} \cdot a_{j k} \cdots a_{u v}
$$

The basic model has been extended to consider two or more older states. A limitation of this type of extensions is the number of parameters that need to be included in the model. A Hidden Markov model (HMM) overcomes this limitation. For a multivariate sequence, a Markov Process could apply to one of the major variables, and the remaining variables are considered to be hidden with regards to the main variable of interest. The hidden variables could be used to estimate the influence of older states on the probability of the next state. In HMM, the probability of a particular sequence could be expressed as a function of the connection between older states and the hidden variables.

Suppose a process that is characterized by a sequence $\left(s_{1}, s_{2}, s_{3}, s_{4}, \cdots\right)$. Every state $s_{i}$ has the following structure $s_{i}=\left(X_{i}, z_{i}\right)$ where $X_{i}$ is one main variable (in this work " $x$ " is each activity) and each $z_{i}$ could be a collection of hidden variables. Then it is possible to characterize the process by the sequence $\left(X_{1}, X_{2}, X_{3}, X_{4}, \cdots\right)$ of the main variables, and a sequence $\left(z_{1}, z_{2}, z_{3}, z_{4}, \cdots\right)$ as their corresponding hidden variables. This means that 
each state " $s$ " will have a main variable " $X$ " and a corresponding set of hidden variables $\left(z_{1}, z_{2}, z_{3}, z_{4}, \cdots\right)$. The probability for a given sequence will be given by

$$
\left.P\left(X_{1}, \cdots, X_{N}, z_{1}, \cdots, z_{N}\right)=P\left(z_{1}\right)\left[\prod_{n=2}^{N} P\left(z_{n} \mid z_{(} n-1\right)\right)\right] \prod_{m=1}^{N} P\left(X_{m} \mid z_{m}\right)
$$

\section{The LSTM component}

In the Long Short Term Memory (LSTM) version of Recurrent Neural Network (Gers, Schmidhuber, and Cummins, 2000), the status of each unit cell in the system is refreshed at every step in a particular manner. Figure 3.4 schematizes this process for an LSTM unit cell. The updating process of each LSTM unit cell is governed by the following set of coupled equations:

$$
\left\{\begin{array}{l}
f_{t}=\sigma\left(U_{f} X_{t}+W_{f} h_{t-1}+b_{f}\right) \\
i_{t}=\sigma\left(U_{i} X_{t}+W_{i} h_{t-1}+b_{i}\right) \\
\tilde{C}_{t}=\tanh \left(U_{C} X_{t}+W_{C} h_{t-1}+b_{C}\right) \\
\theta_{t}=\sigma\left(U_{\theta} X_{t}+W_{\theta} h_{t-1}+b_{\theta}\right) \\
C_{t}=f_{t} * C_{t-1}+i_{t} * \tilde{C}_{t} \\
h_{t}=\theta_{t} * \tanh \left(C_{t}\right)
\end{array}\right.
$$

Here, $C_{t-1}$ : cell state of the previous LSTM cell; $C_{t}$, the resulting state of the current LSTM cell; $h_{t-1}$, the hidden state from the previous LSTM cell; $h_{t}$, the resulting hidden state of 
the current LSTM cell; and $b_{f, i, c, \theta}$, the bias vectors. W's and U's are weight matrices whose values are optimized through the learning process.

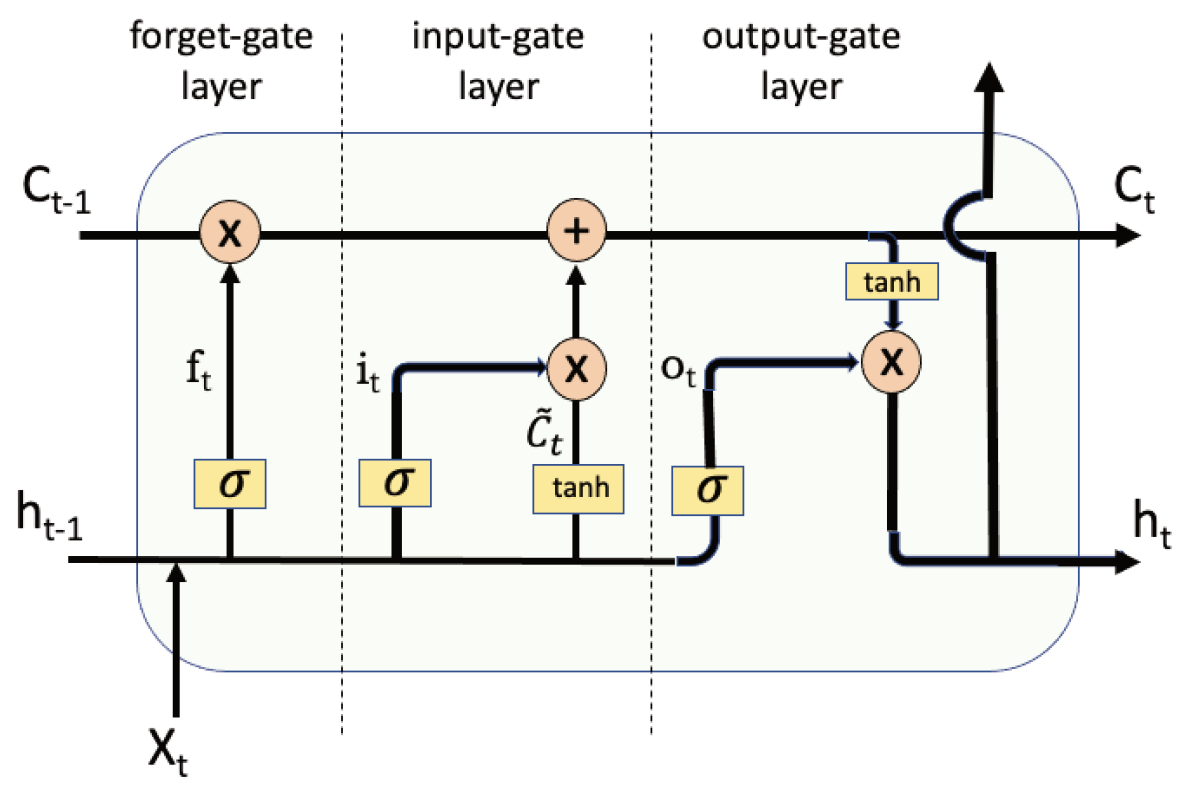

FIGURE 3.4: Internal Mechanism of the LSTM Unit Cell

In the system of equations (1), the forget, the input, and the output gates are represented by $f_{t}, i_{t}$, and $\theta_{t}$, respectively. Inside the LSTM cell unit, the Sigmoid function, $\sigma(z)=\frac{1}{1+e^{-z}}$, is used as the activation function, while the function $\tanh (z)=\frac{e^{z}-e^{-z}}{\left(e^{z}+e^{(}-z\right)}$ is used to generate the cell state candidate, $\tilde{C}_{t}$. The symbol “*” indicates the Hadamard product for matrices, also referred to as the element-wise product. The functioning of the LSTM has been described in detail in (Gers, Schmidhuber, and Cummins, 2000).

The overall process could be summarized as follows. First, in the forget-gate layer, the fresh input data $X_{t}$ and the hidden state from the previous cell are linearly combined using 
a Sigmoid-based operator that is called the activating function. The result is called 'forget gate' because it determines how much information will be irrelevant to update the status of the current cell. Applying the Hadamard product, the forget gate is multiplied by the previous cell state.

Second, in the input-gate layer, a new sigmoid-powered combination, named input gate, is multiplied by a cell state candidate, which is produced by another activating function. In this case, the activating function is a hyperbolic tangent applied over the linear combination of the input and the hidden state of the previous unit cell. This determines which information is relevant enough to be kept by the system. The product of the input gate and the cell state candidate is added to the result of the previous forget layer, producing the cell state of the current LSTM cell unit.

Finally, in the output-gate layer, another sigmoid-powered combination of the input data with the hidden state from the previous LSTM cell is called the output gate. The cell state coming from the input-gate layer is modulated by a hyperbolic tangent function and is element-wise multiplied by the output gate. The result is the new hidden state of the current cell.

The LSTM in this work will be used to predict future values of the hidden variables. In particular, the LSTM model selected is considered as the state-of-the-art technique in this area of research. Here, the LSTM neural network will have up to two medium-sized LSTM layers, each one followed by a dropout layer, plus one dense layer as output layer. In this work, the LSTM component of the model is optimized using Root Mean Square Error (RMSE) and 


$$
R M S E=\sqrt{\frac{1}{N} \sum_{t=1}^{N}\left|y_{t}-\hat{y}_{t}\right|} \text { and } M A E=\frac{1}{N} \sum_{t=1}^{N}\left|y_{t}-\hat{y}_{t}\right|
$$

Mean Absolute Error during its training process. These metrics are expressed as follows:

In both metrics, lower values mean better accuracy and better model performance.

\section{The Classifier component}

The ultimate task of the proposed system is to classify predicted upcoming sequences of states, so that a timely intervention would be required. This classification task will rely on a number of metrics which will estimate the chance of non-regular behavior. These metrics are used as tools for pattern detection.

Let's review how to determine whether a subsequence could be considered an outlier. There are concrete metrics that help to assess whether a particular subsequence should be considered an outlier. Below, common metrics described are "Miss Probability”, "Miss Rate”, and "Entropy Reduction". For a detailed discussion on the metrics, see (Jha, Tan, and Maxion, 2001).

These metrics compare given values from the subsequence and compare them to a threshold to decide if the subsequence present an outlier pattern. The pattern detection process relays on how well a threshold value is defined. The threshold values are parameters that are optimized by the training process. It is expected that all metrics values above the thresholds 
indicate outlier patterns presence.

Miss Probability

For any pair of consecutive transaction states $S_{i}$ and $S_{j}$ in a given subsequence, the indicator $F_{m P}$ is calculated. For a given state $S_{i}$, all the transition probabilities of $S_{i}$ going to any other state within the state space vector are added, except the actual next state $S_{j}$. Then a sum $F$ over all transition pairs in the subsequence and normalize by the number of such pairs is performed,

$$
F_{m P}\left(S_{i}, S_{j}\right)=\sum_{k \neq j} P\left(S_{i}, S_{k}\right)=1-P\left(S_{i}, S_{j}\right)
$$

where $P\left(S_{i}, S_{k}\right)$ is transition probability of going from state $S_{i}$ to $S_{k}$.

Then sum $F$ over all transition pairs in the subsequence and normalize by the number of such pairs,

$$
m s s \text { Prob }=\frac{1}{n_{S e q}} \sum_{i, j \in S e q} F_{m P}\left(S_{i}, S_{j}\right)=\frac{1}{n_{S e q}} \sum_{i, j \in S e q}\left[1-P\left(S_{i}, S_{j}\right)\right] .
$$

Miss Rate

In this metric, the quantity $F_{m R}$ is estimated in a different fashion. For any transition inside the current subsequence, when the transition corresponds to the maximum probability target state, the $F_{m R}\left(S_{i}, S_{j}\right)$ value is set to 0 (zero), otherwise $F_{m R}$ takes the value 1 (one). 
Again, $F$ is summed over all transition pairs in the subsequence and normalize by the number of such pairs,

$$
\text { mssRate }=\frac{1}{n_{S e q}} \sum_{i, j \in S e q} F_{m P}\left(S_{i}, S_{j}\right) .
$$

\section{Entropy Reduction}

For this metric, calculate two quantities $F_{e R}$ and $G_{e R}$, as follow. For a given subsequence Seq, $F_{e R}$ is the entropy for the pair of states under consideration, excluding target state (i.e. all states in the subsequence except for the target in the current pair). In addition, $G_{e R}$ is the entropy for the whole sequence. In other words, $F_{e R}$ is the local, and $G_{e R}$ is the global entropy.

$$
\begin{gathered}
F_{e R}\left(S_{i}, S_{j}\right)=\sum_{k \neq j}-P\left(S_{i}, S_{k}\right) \cdot \log \left[P\left(S_{i}, S_{k}\right)\right] \\
G_{e R}\left(S_{i}\right)=\sum_{k \notin Q}-P\left(S_{i}, S_{k}\right) \cdot \log \left[P\left(S_{i}, S_{k}\right)\right]
\end{gathered}
$$

$F_{e R}$ and $G_{e R}$ are added up over all consecutive state pairs and divide the two sums.

$$
\text { entRed }=\frac{\sum_{i, j \in S e q}\left[\sum_{k \neq j}-P\left(S_{i}, S_{k}\right) \cdot \log \left(P\left(S_{i}, S_{k}\right)\right)\right]}{\sum_{i \in S e q}\left[\sum_{k \notin Q}-P\left(S_{i}, S_{k}\right) \cdot \log \left(P\left(S_{i}, S_{k}\right)\right)\right]}
$$

The pattern detection process will rely on these metrics. The process introduces a sliding window that rolls over the entire sequence with a pre-defined sliding-window-size and determines the size of subsequence to be assesed. It has been reported that using a sliding 
window size (sws) of around 5 states shows good results [7, 23]. Before reaching a conclusion, the results need to be completed with additional analysis and processes.

To distinguish between red and yellow flags, two elements will be considered:

- First, a function will classify a subsequence as positive or negative behavior depending on the final state of that particular subsequence.

- Second, an additional algorithm for subsequence classification will compute a ranking score.

Regarding the metrics and thresholds, an important question is how to choose the right predictor indicator, metrics, and thresholds. Unfortunately, there is no easy answer. Selecting the combination of metrics for the predictor could be left to trial and error, by testing the model performance. A good approach for threshold optimization is to start with a given value for the threshold and refine it iteratively. On the one hand, when the threshold is too low it could result in an increase of the number of false positives. On the other hand, if it is too high, the count of false negatives will increase.

\subsubsection{Model architecture}

As presented in Figure 3.5, first the system loads the data provided by the "Preprocessing module" (which is not part of this work) and prepares the data into ordered sequences of online activities with additional attached features (e.g. time spent in activities, mouse clicks, etc.). Each record has the activity sequence and additional features (e.g. feature 1, feature 2, etc.). Second, a Markov-Chain process is applied to the sequence of activities, and an LSTM is applied to the numerical values of all attached additional features. The additional 
features also feed the HMM. The smart classifier is also fed by the LSTM as well as the MC and/or HMM models.

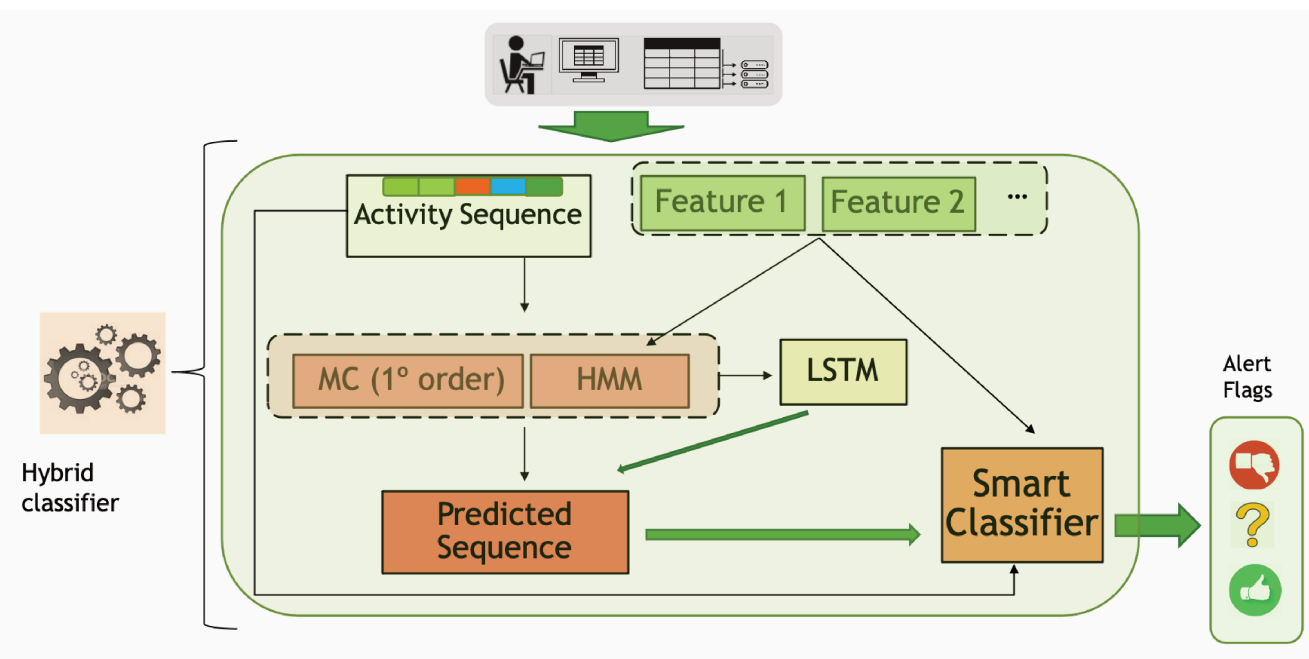

FIgURE 3.5: Detail Technical Design

When the data is loaded into the system, the Hidden Markov Chain component first determines the state space corresponding to the current student's session and, in accordance, the initial probability vector and transition matrix are computed for each variable considered into the HMM. Simultaneously, the LSTM component of the model will be applied to one or more numerical variables from the student's data record selected. The LSTM component will be trained with the initial number of records (number of rows in the data loaded into the system), and then it will provide a forecast for the selected variables with horizon given by the length of the sequence predicted by the HMM component.

The predicted sequence will have a length which is determined by a hyperparameter defined by the Classifier. The sequence will be estimated as follows: a set of random sequences 
is generated and ranked from a weighted combination of the HMM probabilities and the LSTM accuracy prediction.

Predicted sequences, produced by the LSTM forecast output will be assessed by the Classifier, which operates using the metrics for outlier-sequence-detection as it is described in subsection 3.2.3.

\subsubsection{Algorithm design}

In this work, a data review allowed to detect the variables that better describe the student behavior during online lab sessions. All activities were grouped in similar sections to reflect the time devoted to them. Then, for each student, a $\mathrm{M}-\mathrm{C}$ process was developed considering only the activities performed by this particular student.

The system is designed so that a model is developed for each student. Each student has activities registered in six sessions, each with its own re-adjusted model.

This model detects the presence of outliers or anticipate its occurrence. The model has a classifier that estimates scores for a given subsequence. It estimates thresholds to define results for the scores based on a work session. This means that the thresholds define what is considered to be 'normal' for the activity in question, considering the entire history for the active session. 


\section{Input:}

Student online activity logs data, sequential data, numerical and categorical data.

Set of optimized hyperparameters for the Classifier component.

\section{Step-by-step processing:}

Loading the Dataset

Data Scaling

Determining current state space

Building the MC-O(1) and HMM

Update pattern-detection metrics

Select length to train the LSTM predictor component

Last subsequence of the current cycle and the subsequence provided by the LSTM predictor are both assessed by the Classifier.

\section{Output:}

Message or Flag signal to be received by an external sentinel-system module.

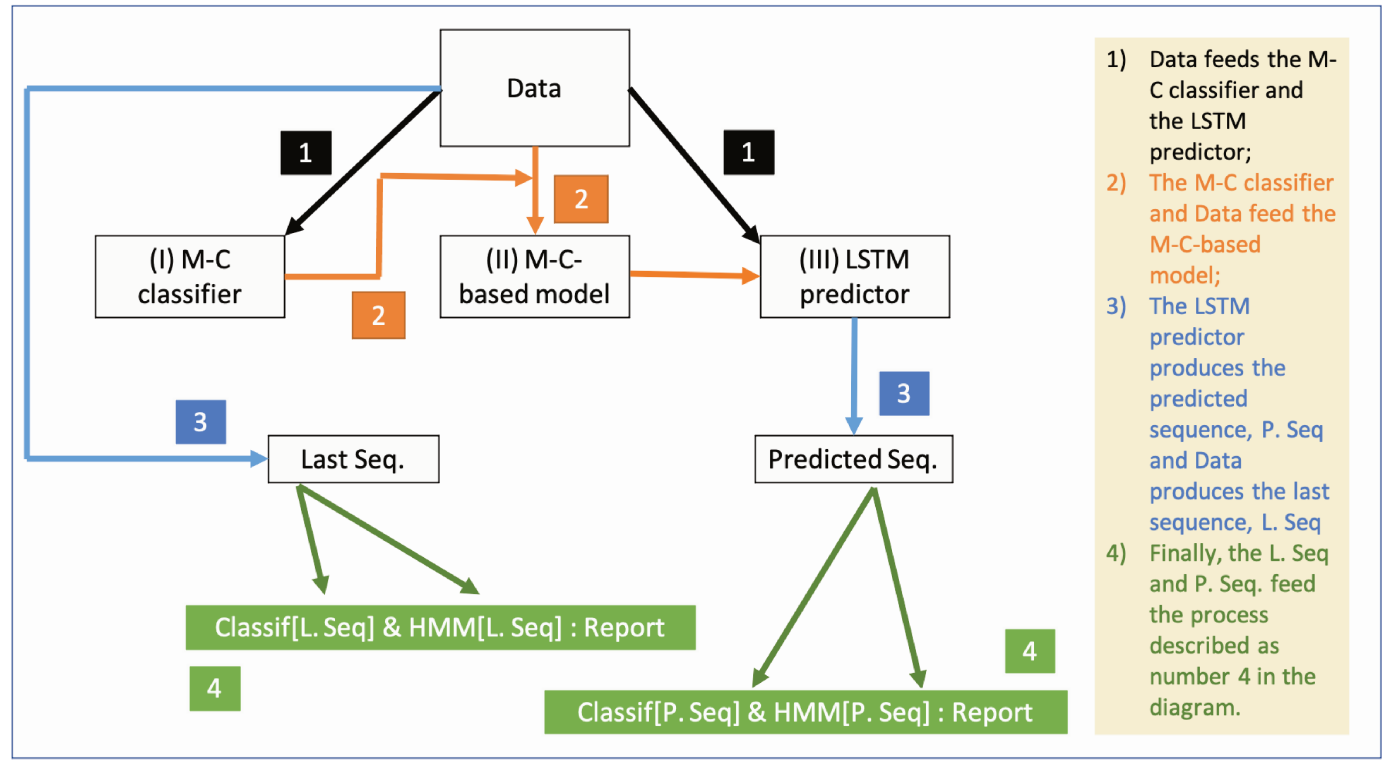

Figure 3.6: Model Flow Chart

The model breaks down activities in chains of short sub-sequences (see Figure 3.6). Predefined metrics on each sub-sequence are estimated. By using a sliding window of the 
same size of each sub-sequence, the model reviews all values for the metrics in each subsequence and compares the values to define a threshold. In this manner, the model builds its classifier, and it also trains its classifier, which means it finds the thresholds of metrics estimates for outlier detection.

The data is processed in real time, this means that the model reads all activities that have entered the data universe up to the present moment, and it builds standards of normal patterns. At the same time, the model trains its LSTM component to predict upcoming sub-sequences in quasi real time. This process is fed by the entire set of data that is available at the time.

A window of recent activities for the last sub-sequence is reviewed and the model predicts the next most likely sub-sequence. Outliers are detected by applying the classifier to both sub-sequences, that is, the last one to occur and the next sub-sequence that has been predicted.

The model builds three metrics, entropy reduction, miss probability and miss rate. By comparing the values of the metrics with its threshold values, the classifier component of the model determines whether a sub-sequence exhibits an outlier pattern. When outlier patterns are detected, the model raises a flag, which could be red, yellow, or green, depending on the combined values of the metrics.

When the entropy reduction is higher than its threshold and at least one of the other two pattern-detection metrics is also above its threshold, a red flag is raised. The yellow flag 
is raised in two instances, one could be when the entropy reduction is above its threshold, but the other two metrics are within normal parameters; or the other one could be when both miss probability and miss rate are above the threshold and the entropy reduction is within its threshold. This is described in table below. All other cases are a match for the green flag, when all three metrics are within threshold, or when the entropy reduction is within threshold, and only one of the other two metrics is above threshold.

The red flag is interpreted as follows: the last sub-sequence read or the next sub-sequence to be predicted are outliers. In this case, the model prints a message. When the flag is yellow or green, the model does nothing at the moment. In the future, however, the yellow flag could also be communicated through an appropriate message.

TABle 3.2: Score Combinations for Flag Activation

\begin{tabular}{lc|c|c|c|c|c|c|c}
\hline \hline & \multicolumn{9}{c|}{ Flags } \\
\cline { 2 - 9 } & \multicolumn{3}{c}{ Red } & \multicolumn{2}{c}{ Yellow } & \multicolumn{3}{c}{ Green } \\
\hline Entropy Reduction & $\triangle$ & $\triangle$ & $\triangle$ & $\triangle$ & $\nabla$ & $\nabla$ & $\nabla$ & $\nabla$ \\
\hline Miss Probability & $\triangle$ & $\triangle$ & $\nabla$ & $\nabla$ & $\triangle$ & $\triangle$ & $\nabla$ & $\nabla$ \\
\hline Miss Rate & $\triangle$ & $\nabla$ & $\triangle$ & $\nabla$ & $\triangle$ & $\nabla$ & $\triangle$ & $\nabla$ \\
\hline \hline
\end{tabular}

Finally, whether or not outliers are detected, the model pauses briefly and restarts its review of history; it refreshes its data universe; and it repeats the process described above. An example of the outlier detection task performed by the model on the Activity sequence of one individual student is presented on Figure 3.7. The scores for determining outliers are trained with all the data from the same student, deciding whether the last subsequence 
or the incoming predicted subsequence are outliers or not, according to all previous subsequences in the session (subsequences with the same length or the same number of Activities stages).

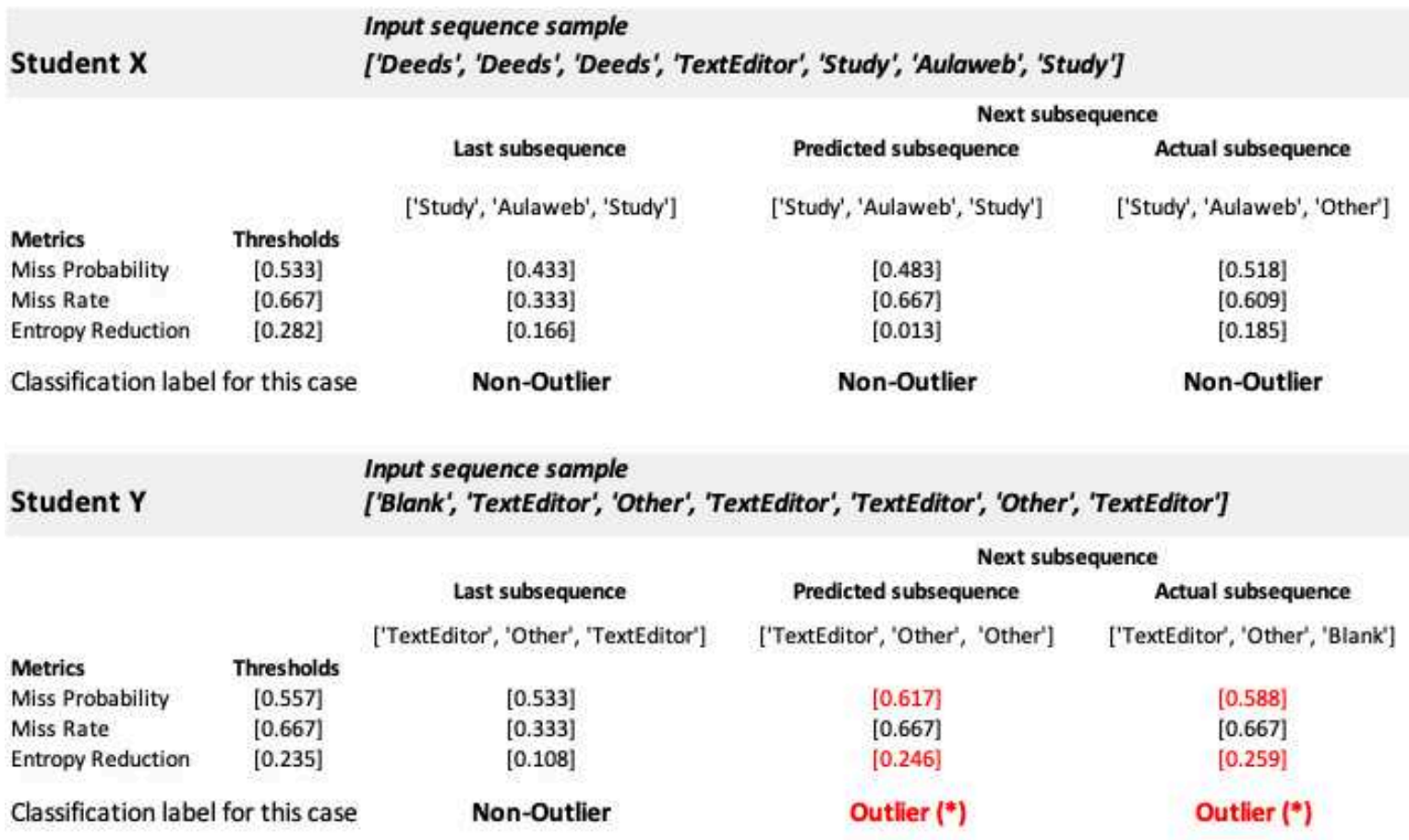

(*) A subsequence labelled as outlier means that at least one of its scores is higher than the corresponding threshold Non-outlier means that none of the scores is higher than its threshold

Figure 3.7: Example of the Outlier Detection Task

\subsubsection{Design discussions}

One important challenge faced by this work is that the system has to be capable of processing incoming data continuously, while, at the same time, it should also continuously deliver opportune outputs in its role as a detection artifact. In order to achieve a competitive performance, the system should be able to perform multiple complex tasks in parallel, and this is addressed in the algorithm design. Another aspect considered in the design is the ability 
of self-adjusting hyperparameters to better cope with time efficiency in the whole process of pattern detection.

\subsection{Evaluation}

This section shows results from experiments that were designed to evaluate the model performance in its capacity for outlier prediction and detection. Based on these preliminary results, the system can be improved in subsequent stages.

\subsubsection{Complexity analysis}

System implementation details Computationally efficiency is one of the main concerns with this type of models. Learning algorithms for sequential supervised learning are highly resource demanding. Some of the limitations of the predictive power of the HMM: Maximum Entropy Markov models (MEMMs), that become more severe with a large number of datasets, will be addressed with the use of neural network, in this case, LSTM.

\subsubsection{Efficiency analysis}

Ongoing experiments assessed the performance of the procedures in terms of computing time. This is presented in the experiments section below. Overall, computing time is acceptable for the type of task. 


\subsubsection{Experiments}

The experiments are designed to evaluate the performance of the system, which is assessed by the accuracy in outlier detection. The following section describes experiments which compare the model results to those closest in the literature.

\section{Predictor module assessment}

Predicting people's behavior is a complex task. One of the reasons could be that people do not behave like machines in terms of performance; so, it is expected that people's behavior will be more difficult to predict compared to machines, as people are guided and influenced by more complex drivers.

Medium stage: One could separate the sub-sequences in three sets: early, medium, and late behavior. Evaluating the predictor at the medium stage could lead to expecting better prediction ability in the medium stages alone compared to the entire sample.

Early and medium: In the entire sample the presence of late erratic behavior tends to lower the scores for the predictor. Another experiment could be to test early and medium stages together and eliminate only the late behavior.

The performance of the predictor as presented in Tables 3.3 and 3.4 is measured in terms of accuracy, right-content, and accuracy in the first predicted state for the incoming subsequence. Average accuracy is defined as the feature of the predicted subsequence by which it fully matches the real subsequence. The right content shows the percentage of the real 
states from the real sequence that can be found in the predicted sequence. Accuracy in the first predicted state for the incoming sequence, as its name indicates, just measures the concordance of the first predicted state with the real first state in the incoming sequence.

In this experiment the classifier component of the model is assessed to understand is quality in terms of its relevance in successfully detecting behavior related to be flagged as outlier. In this experiment one can estimate how many states in the predicted sub-sequence could be linked to a negative behavior in the student.

The classifier thresholds will be estimated across all students for all sessions. For each student, the classifier will apply the global metrics and the metrics specific to each student. With these thresholds, the classifier will be tested as follows:

a) Accuracy: by comparing the scores assigned by the classifier to the predicted subsequence with the scores for the real sub-sequence.

b) Right Content: by finding the percentage of "non-positive" states in the detected outlier sub-sequence. In this case the states "Other" and "Blank" are considered as "non-positive" states. A state is non-positive when its activity is coded as "Blank" or "Other", while positive states are those with activities directly related to the purpose of the lab session.

Adjusting the architecture of the neural network such that this could be efficiently optimized simultaneously for a large number of different datasets with a stochastic nature and sequences of random variables is challenging. The first attempt tries a simple architecture 
TABLE 3.3: Adjusting LSTM Memory Window

\begin{tabular}{lcccc}
\hline \hline & \multicolumn{4}{c}{ Memory Window } \\
\cline { 2 - 5 } Metrics & $\mathbf{5}$ & $\mathbf{7}$ & $\mathbf{1 0}$ & $\mathbf{1 5}$ \\
\hline Accuracy & $\mathbf{3 0 \%}$ & $27 \%$ & $25 \%$ & $23 \%$ \\
Right Content & $\mathbf{4 8 \%}$ & $39 \%$ & $30 \%$ & $26 \%$ \\
$1^{\text {st }}$ State Accuracy & $\mathbf{3 1 \%}$ & $28 \%$ & $29 \%$ & $23 \%$ \\
\hline \hline
\end{tabular}

where accuracy is sacrificed to increase quasi real time processing.

Considering the LSTM architecture used, Table 3.3 displays the results for Session one. It shows that using the best overall behavior is reached for memory window equal to 5 and the percentage of correct prediction for the first state of the sub-sequence, with $49 \%$. For all scores, memory window of 5 outperforms other memory windows tested (Table 3.3 ).

All other experiments are performed with memory window of five, the table below tests results that review data from various sessions, and memory window fixed to 5 .

TABle 3.4: Predictor Module Performance

\begin{tabular}{lcccccc}
\hline \hline & \multicolumn{6}{c}{ Session } \\
\cline { 2 - 7 } Metrics & $\mathbf{1}$ & $\mathbf{2}$ & $\mathbf{3}$ & $\mathbf{4}$ & $\mathbf{5}$ & $\mathbf{6}$ \\
\hline Accuracy & $\mathbf{3 1 \%}$ & $21 \%$ & $18 \%$ & $19 \%$ & $23 \%$ & $20 \%$ \\
Right Content & $\mathbf{4 9 \%}$ & $32 \%$ & $28 \%$ & $27 \%$ & $41 \%$ & $38 \%$ \\
$1^{\text {st }}$ State Accuracy & $\mathbf{3 2 \%}$ & $22 \%$ & $20 \%$ & $24 \%$ & $26 \%$ & $20 \%$ \\
\hline \hline
\end{tabular}


Table 3.4 describes the process of testing the neural network, so that it works for all students. In the case reported, the network architecture has two '100-unit-LSTM' layers. Each one of the LSTM layers is followed by one dropout layer with 0.2 ratio. The architecture closes with a dense layer that is used as an output layer. In the compilation of the model, 150 epochs were used. The memory was 5 stages.

\section{HMM-based metrics}

In this experiment, the assessment focuses on the score of probability, based on the underlying Markov process, that is attached to the predicted sequence. This component should capture the stochastic nature of the student decision.

The HMM probability was combined with the M-C model probabilities to compute the score metrics for the classifier. The thresholds for the classifier were found by estimating all the scores for sequences of 5 stages using a sliding window including all data for all students in the six sessions. This means almost one hundred datasets for each session.

Average thresholds for the three outlier-detection metrics used. For all metrics, both firstorder MC and M-C-based model probability scores are used. With this results for the pattern recognition metrics, the classifier will evaluate each one of the predictions produced by the LSTM predictor for all students across the sessions.

\section{Classifier assesment}


In this type of experiment, the accuracy of the predictor is evaluated, that is, how exact is the predictor at finding the predicted sequence that matches reality. In these experiments, the system compares a predicted sub-sequence to the real values of the next real sub-sequence. When the actual observed values of the sub-sequence are equal to those that had been predicted, the measure of error should be zero.

The accuracy of the predictor component of the model, the LSTM component, is assessed as a percentage of right content or matches in the one sub-sequence predicted when this is compared to real values. This is done for a large number of students. Three matches of five or sixty percent of right predictions is considered a good outcome.

TABLE 3.5: Thresholds Scores for Pattern Detection

\begin{tabular}{lccccccc}
\hline \hline & \multicolumn{7}{c}{ Sessions } \\
\cline { 2 - 6 } Metrics & $\mathbf{1}$ & $\mathbf{2}$ & $\mathbf{3}$ & $\mathbf{4}$ & $\mathbf{5}$ & $\mathbf{6}$ & Avg. \\
\hline Miss Probability (min) & 0.60 & 0.52 & 0.51 & 0.50 & 0.51 & 0.62 & 0.54 \\
Miss Probability (mean) & 0.64 & 0.62 & 0.60 & 0.63 & 0.62 & 0.67 & 0.63 \\
Miss Probability (max) & 0.68 & 0.66 & 0.67 & 0.67 & 0.66 & 0.70 & 0.67 \\
\hline Miss Rate (min) & 0.60 & 0.60 & 0.40 & 0.40 & 0.48 & 0.60 & 0.51 \\
Miss Rate (mean) & 0.66 & 0.61 & 0.60 & 0.63 & 0.61 & 0.73 & 0.64 \\
Miss Rate (max) & 0.80 & 0.80 & 0.67 & 0.67 & 0.66 & 0.70 & 0.67 \\
\hline Entropy Reduction (min) & 0.49 & 0.56 & 0.57 & 0.55 & 0.54 & 0.43 & 0.52 \\
Entropy Reduction (mean) & 0.59 & 0.66 & 0.69 & 0.68 & 0.65 & 0.54 & 0.64 \\
Entropy Reduction (max) & 0.86 & 0.96 & 0.94 & 0.93 & 0.92 & 0.70 & 0.89 \\
\hline \hline
\end{tabular}

Effectiveness, in the tables below, detects whether the label assigned by the classifier to the 
sequence predicted by the LSTM component matches the label that the classifier assigns to the real sequence. The Quality indicators shows how many non-positive states are present in the sequence assigned as outlier. Sensibility captures the percentage of outliers found in each session. With this, the capacity of the system for outlier detection is tuned by the Sensibility measure. This means that with the threshold selection one can determine the sensibility of the system for outlier detection, as it is presented in the tables below. With a minimum average threshold, the number of outliers detected increases considerably, as well as the sensibility percentage.

The classifier thresholds are estimated across all students for all sessions. For each student, the classifier will apply both the global metrics and the metrics that are specific to each student. With these thresholds, the classifier is tested using the standard metrics for classification: Accuracy is the proportion of true results of the total number of cases examined; Precision is the proportion of predicted positives that are truly-positives; Recall (Sensitivity) is the proportion of actual positives that are correctly classified; and F1-Score is the harmonic mean of Precision and Recall. Here both predicted and actual subsequences are considered.

This work uses the following notation. True-positive, $T_{P}$, is used when the positive classification label matches for both the predicted subsequence and the actual subsequence (which means it is an outlier $)$; True-negative, $\left(T_{N}\right)$, is used when the classification label is negative (or non-positive) for both the predicted subsequence and the actual subsequence; False positive, $\left(F_{P}\right)$, is used when the classification label is positive” for the predicted subsequence, but it is negative for the actual subsequence; and False negative, $\left(F_{N}\right)$, is used when the classification label is negative for the predicted subsequence, but it is positive for the actual 
subsequence. The analytic expression for the metrics are:

$$
\begin{aligned}
& \text { Accuracy }=\frac{T_{P}+T_{N}}{T_{P}+F_{P}+F_{N}+T_{N}}, \quad \text { Precision }=\frac{T_{P}}{T_{P}+F_{P}}, \\
& \text { Recall (or Sensivity) }=\frac{T_{P}}{T_{P}+F_{N}}, \quad \text { and } \quad F_{1}=2 \cdot \frac{\text { Precision } \cdot \text { Recall }}{\text { Precision }+ \text { Recall }}
\end{aligned}
$$

In order to assess how the system behaves in terms of the those metrics, two experiments referred as One-Signal configuration and Multi-Signal configuration are carried out. For the One-Signal experiment, Table 3.6, the most restrictive threshold value for each patterndetection metric is used, while for the Multi-Signal experiment, Table 3.7 shows an evaluation of the three characteristic threshold values (max, min., and avg.). The optimized threshold values for the three outlier-detection metrics are listed in Table 3.5. For OneSignal configuration, in Table 3.6, the minimum threshold values were used, while in the Multi-signal configuration, the results reported in Table 3.7, use the minimum, maximum, and mean threshold values.

One-Signal configuration means the system rise the alert when any condition on detectionmetrics is not met.

Observation 1: Results in this One-Signal configuration show that this hybrid approach outperforms other approaches. The solution presented here also allows keep consistency in performance even in early stages of the detection task, when log data is not very large.

Observation 2: Performance metrics score better in the intermediate sessions. One reason for this could be that users tend to have steadier behavior patterns in those sessions. In contrast, in both initial and closing sessions there are some factors such as learning curve 
with the platform (in the initial session), and perhaps in the closing session, users experience certain stress related to the need of timely finishing the session's duties because it is the last session.

TABle 3.6: One-Signal Configuration

\begin{tabular}{lccccccc}
\hline & \multicolumn{7}{c}{ Session } \\
\cline { 2 - 6 } Metrics & $\mathbf{1}$ & $\mathbf{2}$ & $\mathbf{3}$ & $\mathbf{4}$ & $\mathbf{5}$ & $\mathbf{6}$ & Average \\
\hline Accuracy & 0.77 & 0.87 & 0.82 & 0.87 & 0.85 & 0.79 & 0.83 \\
Precision & 0.80 & 0.90 & 0.86 & 0.92 & 0.92 & 0.92 & 0.89 \\
Recall (Sensibility) & 0.89 & 0.95 & 0.93 & 0.93 & 0.92 & 0.84 & 0.91 \\
F1 Score & 0.85 & 0.93 & 0.89 & 0.93 & 0.92 & 0.88 & 0.90 \\
\hline \hline
\end{tabular}

Observation 3: In session 5 (Table 3.6), the number of false negatives equals the number of false positives, resulting in the equality of Precision, Recall, and F1 scores.

Multi-Signal configuration means the system rise the alert when a combination of more than one conditions on detection-metrics are not met.

One of the main objectives of the system designed here is to carry out a pattern-detection task that could anticipate odd behavior early enough. The percentage of effectiveness should show this feature, that is how early in the process one could detect outlier patterns. The effectiveness percentage tends to be higher with the maximum threshold when only a few outliers have been detected. The usefulness of a potential system based on this 
model could be related to these criteria.

TABLE 3.7: Multi-Signal Configuration

\begin{tabular}{|c|c|c|c|c|c|c|c|c|c|c|c|c|}
\hline \multirow[b]{2}{*}{ Metrics } & \multicolumn{3}{|c|}{ Session 1} & \multicolumn{3}{|c|}{ Session 2} & \multicolumn{3}{|c|}{ Session 3} & \multicolumn{3}{|c|}{ Averages } \\
\hline & $\operatorname{Max}$ & Mean & Min & $\operatorname{Max}$ & Mean & Min & $\operatorname{Max}$ & Mean & Min & $\operatorname{Max}$ & Mean & Min \\
\hline Accuracy & 0.727 & 0.649 & 0.623 & 0.610 & 0.476 & 0.781 & 0.552 & 0.483 & 0.747 & 0.578 & 0.523 & 0.706 \\
\hline Precision & 0.143 & 0.222 & 0.608 & 0.194 & 0.357 & 0.894 & 0.119 & 0.359 & 0.808 & 0.127 & 0.319 & 0.776 \\
\hline Recall & 0.500 & 0.500 & 0.775 & 0.462 & 0.484 & 0.843 & 0.714 & 0.633 & 0.881 & 0.473 & 0.583 & 0.834 \\
\hline \multirow[t]{2}{*}{ F1 Score } & 0.222 & 0.308 & 0.681 & 0.273 & 0.411 & 0.868 & 0.204 & 0.458 & 0.843 & 0.196 & 0.410 & 0.802 \\
\hline & \multicolumn{3}{|c|}{ Session 4} & \multicolumn{3}{|c|}{ Session 5} & \multicolumn{3}{|c|}{ Session 6} & \multicolumn{3}{|c|}{ Averages } \\
\hline Metrics & $\operatorname{Max}$ & Mean & Min & $\operatorname{Max}$ & Mean & Min & $\operatorname{Max}$ & Mean & Min & $\operatorname{Max}$ & Mean & Min \\
\hline Accuracy & 0.535 & 0.515 & 0.748 & 0.604 & 0.560 & 0.692 & 0.441 & 0.452 & 0.643 & 0.578 & 0.523 & 0.706 \\
\hline Precision & 0.073 & 0.296 & 0.798 & 0.132 & 0.360 & 0.766 & 0.100 & 0.320 & 0.781 & 0.127 & 0.319 & 0.776 \\
\hline Recall & 0.273 & 0.615 & 0.893 & 0.625 & 0.692 & 0.855 & 0.267 & 0.574 & 0.758 & 0.473 & 0.583 & 0.834 \\
\hline F1 Score & 0.115 & 0.400 & 0.843 & 0.217 & 0.474 & 0.808 & 0.146 & 0.410 & 0.769 & 0.196 & 0.410 & 0.802 \\
\hline
\end{tabular}

Then, in order to assess the usability of the Smart-Classifier model presented in this work, it is tested in terms of:

Effectiveness - by comparing the scores assigned by the classifier to the predicted subsequence with the scores for the real sub-sequence.

Perceptiveness - by finding the percentage of 'non-positive' states in the detected outlierpattern subsequence. In this case, above mentioned, the states 'Other' and 'Blank' are considered as 'non-positive' states. states (students are engaged on activities that are not meant to be carried out during the current session, say, for example, students using social media, 
youtube, this is labeled as 'others').

in other words, Effectiveness is, the total matching cases in label assignation between the predicted and the real incoming subsequence divided by the total number of predicted subsequences. Let be $N_{m E}$ the total number of events in which, after the prediction, with Label[predicted subsequence] $=\operatorname{Label}\left[\right.$ actual subsequence], and $N_{p}$, the total number of predictions made, then: $\quad$ Effectiveness $=\frac{N_{m E}}{N_{p}} \times 100=$ Accuracy $\times 100$.

This is a percentage of correct label assignation to the predicted subsequence (whether it be an outlier or not). In other words, overall how well the classification task is performed. By contrast, Perceptiveness is the total matching cases of predicted and real subsequences containing over $60 \%$ of states (which are activities "blank" and "other"), over the total number of predicted subsequences. Let be $N_{m P}$ the total number of events in which, after the prediction, then if the percentage of states "blank" and "other" in [predicted subsequence] $>60 \%$, and also the percentage of states "blank" and "other" in [actual subsequence] $>60 \%$. Then, this perceptiveness rate is computed as: $\quad$ Perceptiveness $=\frac{N_{m P}}{N_{p}} \times 100$.

This means that, even if the system does not detect such subsequences as outliers (according to the session history of the particular student), the system is still able to detect that the entire sequence could have problems.

By analyzing the achieved results, it can be observed from Table 3.8 that, using a more restrictive threshold increases the Perceptiveness of the system, as presented in the results. But on the contrary, with less restriction, it is Effectiveness that improves. Taking the average mean threshold shows better scores, but this scenario does not perform well as a 
classifier as evidenced in the previous experiment.

TABLE 3.8: Smart-Classifier Usability

\begin{tabular}{|c|c|c|c|c|c|c|c|c|c|}
\hline \multirow[b]{2}{*}{ Criteria } & \multicolumn{3}{|c|}{ Session 1} & \multicolumn{3}{|c|}{ Session 2} & \multicolumn{3}{|c|}{ Session 3} \\
\hline & $\operatorname{Max}$ & Mean & Min & $\operatorname{Max}$ & Mean & Min & $\operatorname{Max}$ & Mean & Min \\
\hline Effectiveness & $73 \%$ & $61 \%$ & $44 \%$ & $61 \%$ & $48 \%$ & $40 \%$ & $55 \%$ & $45 \%$ & $39 \%$ \\
\hline \multirow[t]{2}{*}{ Perceptiveness } & $0 \%$ & $33 \%$ & $69 \%$ & $0 \%$ & $20 \%$ & $64 \%$ & $0 \%$ & $50 \%$ & $54 \%$ \\
\hline & \multicolumn{3}{|c|}{ Session 4} & \multicolumn{3}{|c|}{ Session 5} & \multicolumn{3}{|c|}{ Session 6} \\
\hline Metrics & $\operatorname{Max}$ & Mean & Min & $\operatorname{Max}$ & Mean & Min & $\operatorname{Max}$ & Mean & Min \\
\hline Effectiveness & $54 \%$ & $48 \%$ & $45 \%$ & $60 \%$ & $54 \%$ & $32 \%$ & $45 \%$ & $44 \%$ & $44 \%$ \\
\hline Perceptiveness & 0 & $33 \%$ & $47 \%$ & $0 \%$ & $14 \%$ & $47 \%$ & 0 & $67 \%$ & $62 \%$ \\
\hline
\end{tabular}

Finally, the chart in Figure 3.8 shows a comparison with related work mentioned on Table 2.1. Results show that the hybrid approach presented here (combining Markov-Chain based model with Recurrent Neural Networks), outperforms other approaches reviewed in the literature. Another key observation is that the data size in this work is smaller compared to related work, which means that the space for improvement is still very large.

The bottom line here is that, all scores are very good when compared with all related work in Table 2.1. It is important to note that the accuracy and precision scores are as good as those found in related work, even when the data for his research does not have the adequate size of data, that is, there is no a well-established ground-truth available in . 


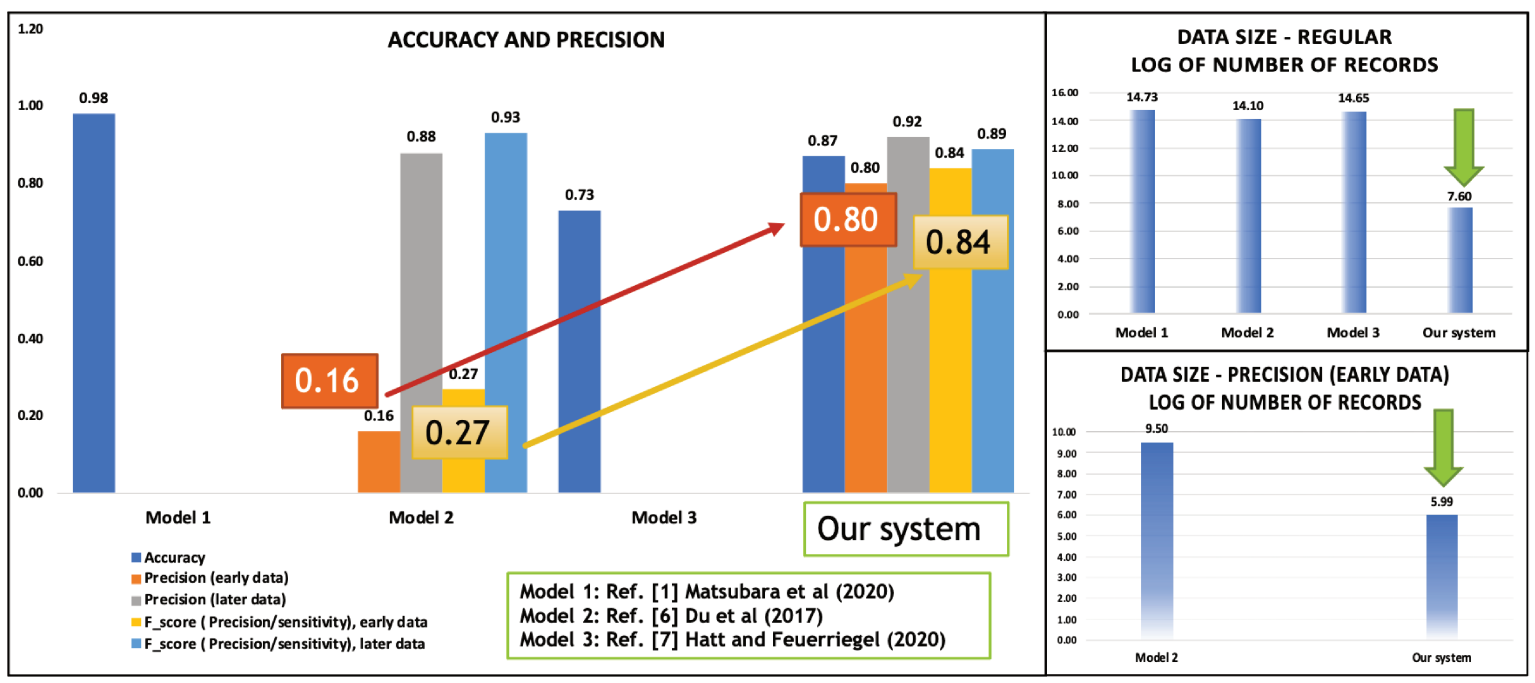

Figure 3.8: Performance Comparison

In related work discused in Chapteer 2 it can be seen accuracy reports scoring $0.98,0.65$, and 0.73 . In one of the papers there i reported a precision score of 0.16 (in similar conditions with limited data, i.e. early stages of the running process) and 0.90 when sufficient data is available. In this work, as it can seen on Figure 3.8 all the best average scores are as good or better than related work. The average accuracy in this work is 0.71 , (but some individual values are higher at 0.78 ). In precision, this work presents individual values of up to 0.89 , with average score of 0.78 .

\subsection{Discussion}

The predictor module is based on an LSTM-RNN architecture that is fed with non-homogeneuos data, including categorical data. Considering its contribution to the overall system performance, it behaves relatively well. However, a few observations about its performance can be discussed in more details. 
It is important to note that the specific challenges that arise from the processing of nonhomogeneuos data through a neural network cannot be ideally handled with a single fit-all approach. Categorical data has to be converted to numerical data so that the neural network can handle it appropriately. Choosing the encoding method that fits the perspective of the selected approach usually comes with a tradeoff in performance.

A key idea in this research is to facilitate interpretability of the model outcomes. Following this line of thought, there is an underlying process driving the data flow that is intended to be modelled in a point-process-like fashion. The Markov-Chain approach used addresses this goal. Using the ordinal encoding, each value of the categorical target variable is assigned to an integer number. To avoid the ordinal relationship that the ordinal encoding imposes, unique values for the target variable were ranked according to their frequency and considering all available data. This allows to transfer the underlying probabilistic nature of the values in the sequence to the neural network. Although this approach offers reasonable good behaviour when the frequency distribution is known, its reliability is very limited when the probability distribution of the new data is unresolved. This is a weakness of this approach at the current stage. Using one-hot encoding, potential problems related to the ordinal relationship are avoided, specially when the frequency distribution of the variable to encode is difficult to estimate. In this scenario, HMM probabilistic scores are included to feed the neural network, as well as to provide the neural network (the predictor module) with some insight about the perspective to be introduced through all the model components.

Ordinal encoding with labels for the target values ranked by their frequency would be the 
most appropriate method, considering that the aim is describing the evolution of the clickstream data as a point-process-like model. However, the lack of sufficient data to ensure stability in frequency distribution renders the adoption of one-hot encoding more suitable, despite limitations on interpretability. In the next stage of the research, a Bayesian graph network model is introduced to address this limitation. 


\section{Chapter 4}

\section{Transformer with Modified Attention}

\section{Mechanism}

The need for quasi-real time feedback and the characteristics of the limited data generated in online activity, require better predictive capacity. The work in this chapter contributes to improve the predictive capacity of the predictor module of the Smart Classifier developed in the previous chapter, which is progressively fed by somewhat erratic and small data generated at the early stages of the interaction with LMS. The approach in this work facilitates customizations of the attention mechanism in Transformer models.

\subsection{Predicting User Decision with Clikstream Data}

In this research, the prediction problem in categorical sequential data is addressed, and how clickstream data could leverage performance when the target variable can be described as highly stochastic. A solution to this problem explored in this paper is a model that combines a Bayesian Graph Network with a modified Sequence-To-Sequence Model based on 
an Encoder-Decoder system powered by Long Short-Term Memory (LSTM) / Gated Recurrent Unit (GRU) neural networks.

This work focuses on building a model to predict categorical sequential data with highly random stochastic components. In the solution presented, clickstream data will be used to improve the performance of the model. Clickstream data is associated to every stage of the sequential data or time steps.

The Transformer model, an upgrade of encoder-decoder models based on LSTM/GRURNNs, is one of the most successful approaches in sequential data prediction. The key feature with Transformer models is the attention mechanism introduced in encoder-decoder architectures (Vaswani et al., 2017), (Luong, Pham, and Manning, 2015).

The role of the attention mechanism in Transformer is to maximize short-long dependencies in portions of the next-to-be-processed input data and to understand the context of those dependencies that leads to the next prediction output. In other words, the attention mechanism makes an active monitoring to decide when and how to adjust the weights of relevant dependencies in portions of the data during the training process to improve the forecasting output. Therefore, in this picture, with "context" we are talking about the dependencies that can play a key role in a portion of the data, that could be specific only to that data portion.

Transformer model performs very well in Natural Language Processing (NLP) problems, especially for such applications as language translation, language modelling, audio-to-text 
conversion, among others (Wu et al., 2020). Despite its unquestionable success in many applications, Transformer models still have some limitations.

On one side, in Transformer models, computational task is increased quickly due the addition of more weight parameters. As it has been indicated in the context of a sequenceto-sequence model (e.g. RNN-based encoder-decoder) (Sutskever, Vinyals, and Le, 2014), attention is a very effective mechanism because the context vector contains the most relevant information from the encoder. The attention mechanism works by combining the encoder's output at each time step, with the decoder's output at time step $t$, to generate the context vector for that time step. When the input data for the model are long sequences, this procedure greatly increases training time (Luong, Pham, and Manning, 2015).

On the other, in problems such as NLP it is not so difficult to extract some meaning from portions of the data in a way that it allows to establish a sort of mapping to elaborate a meaningful output. In those cases, the ideal mapping tool is a dictionary. In other cases, for example in DNA sequencing problems, any helping tool came from biochemical interactions (Shadab et al., 2020). When the predicting problem is dealing with categorical sequences that could evolve as a stochastic variable, the finding of a suitable mapping tool is difficult. This could be the case when the target variable depends on personal preferences and no ground truth is available (Amezaga and Shafiq, 2021a).

The approach in this work takes another direction, to use clickstream data as a source of context for the portion of data to be processed. The clickstream data will feed the modified attention mechanism in the model by creating a scoring index, based on joint probability 
of a BGN model (Schoot et al., 2021) which is associated to every time-step for the categorical target variable. The evolution of this Bayesian scoring in each subsequence feeding the network will provide additional information that could help the sequence-to-sequence model to learn a meaningful context to improve predicting capability.

In summary, the problem addressed is concerned with how a hybrid solution combining a BGN with a Transformer model could leverage performance in categorical sequence prediction problems for stochastic data. The research question that stem from this stage of the work are:

RQ: How data processing using a BGN could enhance performance in a sequence-tosequence forecasting engine based on a Transformer?

\subsection{The Modified Transformer}

The goal is to build an encoder-decoder system to be powered by a BGN modeling the clickstream time-evolution. A graph network is used to compute a score associated to every time-step of the sequential data. This score will be used to be inserted in the attention mechanism for the corresponding transformer model.

The methodology could be summarized as clickstream data, associated to the categorical target variable, used to build a BGN. This BGN computes a scoring value to each state of every subsequence of the input sequence. The length of each subsequence is a hyperparameter of the model to be optimized, so that an appropriate balance between accuracy 
and speed could be achieved as part of the overall performance of the model.

The Transformer Model uses the Bayesian scoring values to better analyze the "context" of every single state in a subsequence. The calculated Bayesian scoring indexes will be added to the attention layer as a modulator over the encoder output and the decoder output before combining both in the training process.

Finally, a new subsequence is predicted. At this stage of the work, the aim is to build a forecasting engine that is suitable for sequential categorical data. The system is powered by a graph Bayesian network model and a recurrent neural network. When the categorical sequence data is generated by an underlying stochastic process, an extra guide is needed to help the system to understand the "context", so that a given input subsequence could predict the next subsequence.

In the proposed approach, the BGN, fed by clickstream data, modifies the attention mechanism in a Transformer architecture. The inclusion of the clickstream data could leverage performance, while helping in predicting problems that involve categorical sequential random variables.

\subsubsection{Overall process}

The data sequences and additional information are gathered from a collecting system and processed dynamically by the hybrid smart classifier, which generates alert flags (i.e. positive, negative, or anomalies). The flags activate an alert assistant that provides an informed 
feedback to the user, or to an assistant who timely will provide meaningful tips and recommendations to the user. This process is sketched in Figure 4.2.

\subsubsection{Data description}

Data on user online activity will be used to test the potential of this method for uncovering hidden relationships among desired data features that may indicate the probability of success in a particular set of online activity strategies.

Data on online activity from around one hundred users, from student 1 to student 115 is collected (Vahdat et al., 2015). A selection of features included in the data is shown on Figure 3.3. In theory all users engaged online in six work sessions, $w_{1}$ to $w_{6}$, to complete one laboratory assignment and obtain one final grade, with the highest grades indicating success and the lower grade or absence of records indicating failure. Some users failed to participate in some of the work sessions, but in principle, each user would have had the opportunity to complete the six work sessions. For each user $s$, each work session $w$ produced a set of records, from $r_{1}$ to $r_{M}$.

\section{Data Structure}

The focus of the analysis is on any given record $r$, which could also be defined as a sequence of activities and features. For each user $s$ and each work session $w$, each record $r$ is a matrix or set of time series with time $t_{1}$ to $t_{F}$. The time series in each user record $u_{i}$ are:

- the activity type, which has a string format and is one character identified with a letter, there are about nine activity types; 
- the duration of the activity in seconds, which is a numeric format time series; and

- about four additional time series that define up to four additional features.

\subsubsection{System model}

The solution is compound by a BGN with state-to-state transitions governed by an underlying Markov-Chain process, and an LSTM-based Transformer model.

\section{The Bayesian Graph Network}

As it has been documented above, a component of the model in this work is a Markov-Chain $(\mathrm{MC})$ process. In the $\mathrm{MC}$ process, the system is a sequence with $N$ elements or states. Each state belongs to the state space vector $Q$, the set of all possible states accessible to the system.

Let $Q=\left\{q_{1}, q_{2}, \cdots, q_{r}\right\}$ be the state space vector of the system. In this work, $Q$ is the set of all different activities a user could be working on during a session, this means, each $q_{i}$ denotes an activity available to the user.

The components of the initial probability vector are the frequencies of each component of the state space vector, $\vec{\Pi}=\left\{\pi_{1}, \pi_{2}, \cdots, \pi_{r}\right\}$, where $\pi_{i}=P\left(s_{0}=q_{i}\right)=\frac{1}{N} \times$ [total count of $\left.q_{i}\right]$.

All user transitions from an activity to another will be ruled by the Markov Chain transition matrix, $\mathbf{A}=\left\{a_{i j}\right\}$, where $a_{i j}=P\left(s_{t}=q_{j} \mid s_{t-1}=q_{i}\right)$. 
A BGN is built using the sequential data for the activities, and the click stream data associated to them. It is assumed that an underlying Markov Chain Process (Schoot et al., 2021) will drive the user selection of which activity to work with next. Formally, a Bayesian network is a directed acyclic graph, whose nodes represent a set of random variables $X_{1}, \ldots, X_{n}$; the joint distribution of these variables can be written as

$$
P\left(X_{1}, \ldots, X_{n}\right)=\prod_{i} P\left(X_{i} \mid P_{k}\left(X_{i}\right)\right)
$$

where $P_{k}\left(X_{i}\right)$ is a set of parents of $X_{i}$.

The Bayesian joint-probability of the user will be transitioning from the activity $A_{i}$ at timestep $t$ to the activity $A_{t}$ at time-step $t+1$. For each node of the BGN there are a number of click data associated to the user activity and the duration in the activity $(D t)$. The focus is on two of the most relevant features of the click data, "keystrokes" (KyS) and left-mouse $\operatorname{clicks}(\operatorname{LmC})$.

$A_{t}$ is used as notation for any activity $\left\{A_{1}, A_{2}, \cdots, A_{n}\right\}$ at time-step $t$, and it could be any activity from the space state.

- Dt: time spent in a particular activity by the user.

- KyS: Keystroke hit by the user during the particular activity $A_{t}$.

- LmC: Left-mouse clicks by the user while in activity $A_{t}$.

Each time-step, the user is in one of the activities accessible to her/him, and the amount of $K y S, L m C$, and other click data is recorded along with the time spent in the activity. The 
set $\{A\}$, that is the whole sequence of categorical values will be considered as latent variable of the model, while the click stream data represent the observable data in this model. A BGN is built using the sequential data for the activities, and the click stream data associated to them. The purpose of the BGN component in the solution is to compute a scoring index for each activity chosen by the user from the set of available activities (the state space). The index will depend on the Bayesian joint-probability $P\left(A_{t}, K y S, L c M\right)$.

\section{Encoder-Decoder Systems}

For problems where both input and output are sequences, a sequence-to-sequence (Seq2Seq) machine-learning architecture could be proposed. In general, the sequence-to-sequence could also be described as an encoder-decoder system. Overall, this type of model works as follows. First, a stack of RNNs or CNNs, the encoder, captures relevant structures from the input sequence and creates a context vector. Second, the context vector is transferred to the decoder, which is another stack of RNNs layers. Finally, the decoder connects the information contained in the context vector with the corresponding output sequence.

\section{Attention Mechanism}

An attention mechanism is a feature that could be added to an encoder-decoder system (Vaswani et al., 2017). This allows to better understand context in input sequence. A set of key-value pairs and a query could be linked to an output. When the keys, values, query and output are all vectors, the link between them is called an attention function. An individual weight of each value is estimated by the compatibility function of the query with its 
matching key; and the output is a weighted sum of the values. An encoder-decoder system that features an attention function is called a Transformer. In the models an LSTM or GRU RNNs are used as core components of both the encoder and the decoder. The figure (4.1) features the internal structure of an GRU recurrent unit cell.

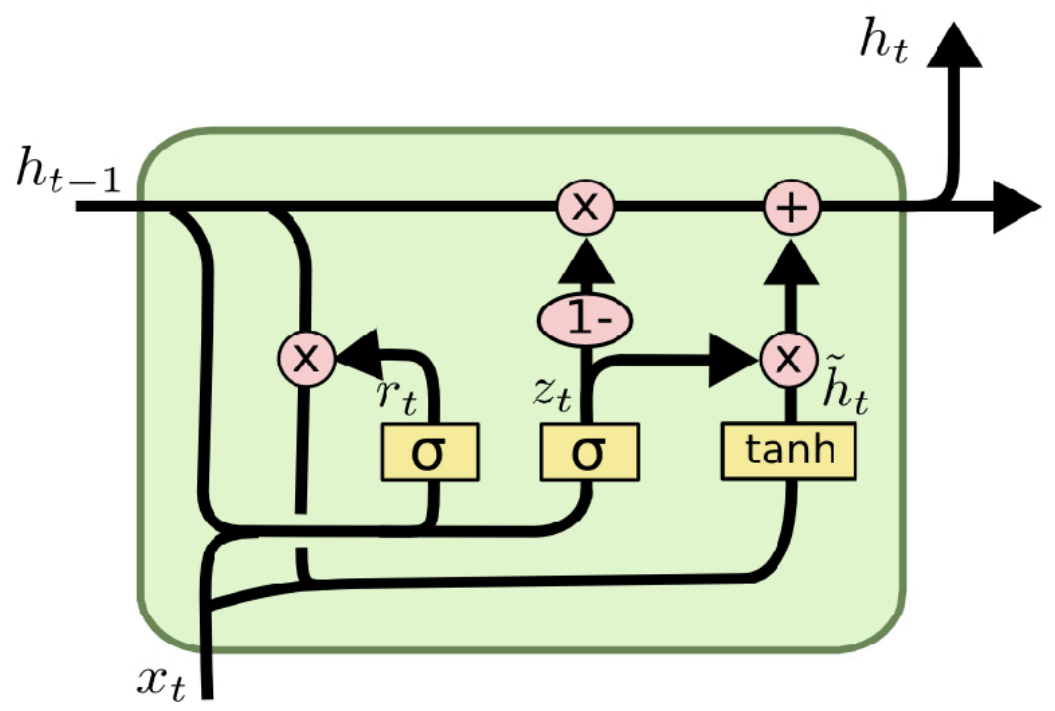

FIGURE 4.1: GRU Cell Unit

Very much like LSTM units, the Gated Recurrent Unit (GRU) is also an RNN architecture. In this case, rather than the input, output, and forget gate of the LSTM, the GRU has the reset gate and the update gate. The way the new input is combined with the previous memory info is set on by the reset gate, while how much of the previous memory to keep is defined at the update gate. In (4.1) the reset gate is represented by $r_{t}$, and the update gate by $z_{t}$. 


$$
\left\{\begin{array}{l}
z_{t}=\sigma\left(U_{z} x_{t}+W_{z} h_{t-1}\right) \\
r_{t}=\sigma\left(U_{r} x_{t}+W_{r} h_{t-1}\right) \\
\tilde{h}_{t}=\tanh \left(U_{h} x_{t}+W_{C}\left(r_{t} * h_{t-1}\right)\right) \\
h_{t}=\left(1-z_{t}\right) * h_{t-1}+z_{t} * \tilde{h}
\end{array}\right.
$$

In systems of equations (3.1) and (4.1), the forget, the input, and the output gates are represented by $z_{t}$, and $t$, respectively. Inside the GRU cell unit, the Sigmoid function, $\sigma(z)=\frac{1}{e^{z}+e^{-z}}$, is used as the activation function, while the function $\tanh (z)=\frac{e^{z}-e^{-z}}{e^{z}+e^{-z}}$ is used to generate the cell state candidate, $\tilde{h}_{t}$. The symbol $*$ indicates the Hadamard product for matrices, also referred to as the element-wise product.

\subsubsection{Architecture}

In Chapter 3, a system is developed to predict a new sequence of online activity for users, e.g. students working online. First, a Markov-Chain (MC) model reads the entire activity history for one student, and trains an Inspector module. At the same time a predictor module, in this case a Long Short Term Memory or LSTM, also reads the student's history of online activity and predicts the New Sequence, that is the next sequence of steps. Second, the trained Inspector module evaluates both the Last Sequence (LS) and the predicted new sequence. Finally, the trained Inspector module launches a label to conclude the process.

In this work, as presented in Figure 4.2, first the system loads the data provided by the "Pre-processing module" and prepares the data into ordered sequences of online activities 
with additional attached features (e.g. time spent in activities, mouse clicks, etc.).

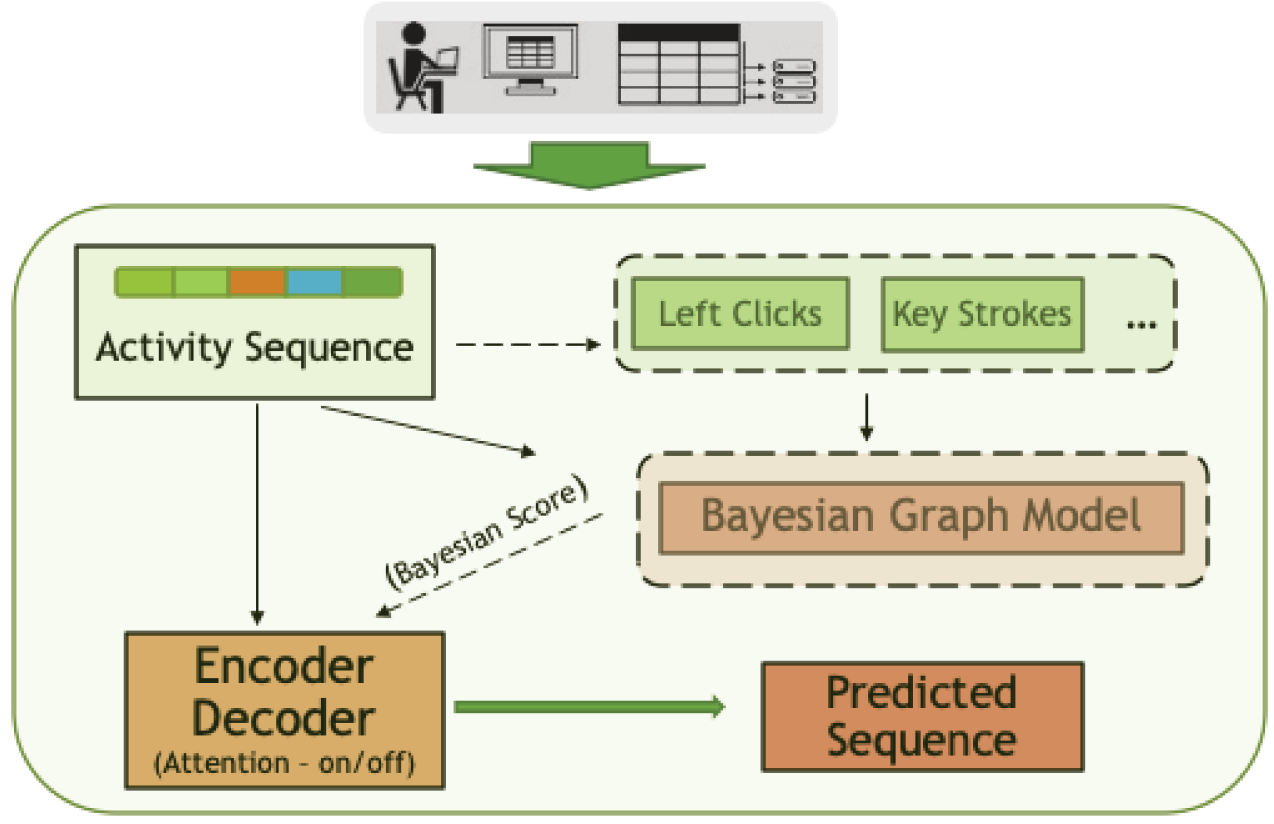

Figure 4.2: Predictor Component Flow Chart

The recorded clickstream data is used to build a BGN that computes a scoring. This Bayesian scoring helps to analyze context for the prediction process. This score feeds the attention mechanism of a Transformer model for the predictor module. The focus is on improving the performance of the predictor module that creates the New Sequence (NS). In this approach, the predictor module is designed as a Sequence-to-Sequence model with an Encoder-Decoder architecture. Its improvement is done in several steps.

In a first version of the Sequence-to-Sequence model, both the Encoder and the Decoder are feed-forward LSTMs. In a second version of the model, the Encoder has bidirectional LSTM 
layers and the Decoder has feed-forward LSTM layers. In the third version, the Sequenceto-Sequence model is built as a Transformer model. The multiplicative attention (Loung Attention) layer is applied in the Encoder-to-Decoder transition.

Finally, in the fourth and last version, the model will be a Hybrid Ensemble containing a BGN model coupled to a Transformer. The BGN is added to the Transformer model modifying its attention mechanism. This represents a substantial change to the standard treatment of the attention mechanism in Transformers found in the literature. This is a new approach for the analysis of the relevant context in the forecasting of multi-variated and non-homogeneuos sequential data.

In this approach, the BGN is a model that represents how to estimate the next activity based on the previous one. The activities are considered as latent or hidden variables and the estimation process is based on scores that are computed through the observable variables, which are clickstream data and the time length of each activity.

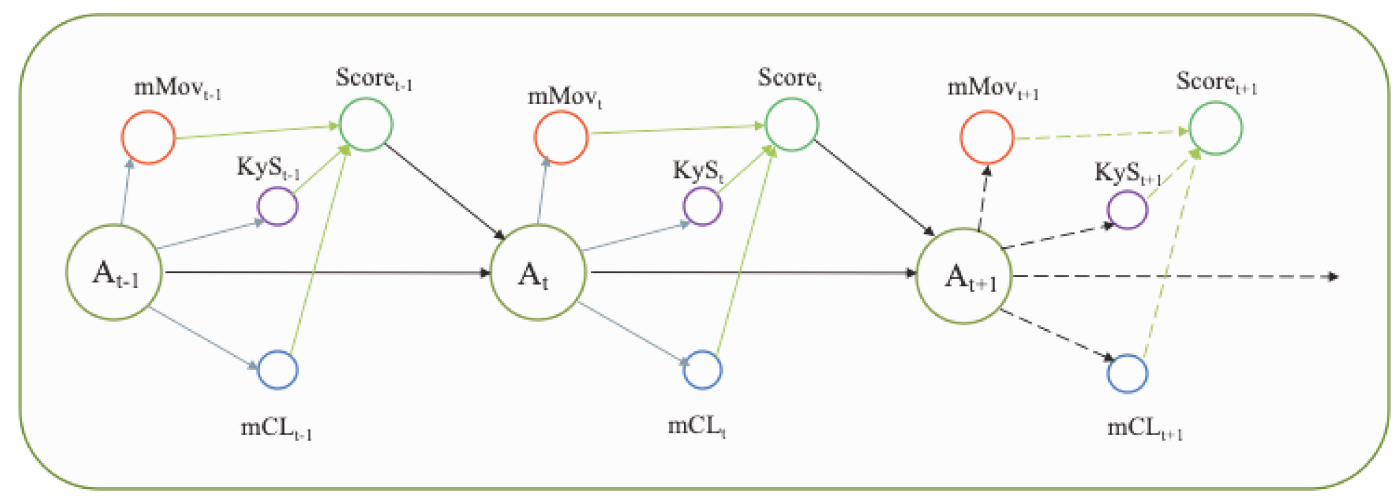

FIGURE 4.3: Bayesian Graph Network

In this work, the BGN purpose is to estimate a Bayesian score to be used in the attention 
mechanism (AM) of the Transformer Model (TM). The Bayesian score is included in the computation of the alignment score, before the context is estimated. In Figure 4.3 the BGN diagram shows this process.

The BGN model will compute scores, that is, probabilities for each incoming sequence to compute the score alignment. Here, the Bayesian Behavior Scoring is computed as

$$
S_{B G N}\left(A_{t}\right)=P\left(A_{t} \mid A_{t-1}\right) \cdot \exp \left[\alpha \cdot P\left(m C L_{t} \mid A_{t}\right)+\beta \cdot P\left(K y S_{t} \mid A_{t}\right)-\delta \cdot m M o v_{t}\right]
$$

Where $m C L$ is left mouse-clicks; KyS is key strokes; $A$ is Activity (state), and $m M o v$ is a proxy for the elapsed time in the activity. Hyperparameters in equation (4.2) $\alpha, \beta$, and $\delta$ are meant to adjust the BGN model.

Each row of clickstream data as presented in Figure 4.4 shows the observable variables, such as left mouse-clicks, key strokes, and mouse movement. The observable indicators are processed by the BGN. The Bayesian scoring is transferred to the Transformer model. Then, the output sequences are generated.

\subsubsection{Algorithm design}

In this work, a data review allowed to detect the variables that better describe the user behaviour during online sessions. The system is designed so that a model is developed for each user. Each user has activities registered in several sessions, each with its own re-adjusted model. The Ensemble is implemented in Python language programming using 


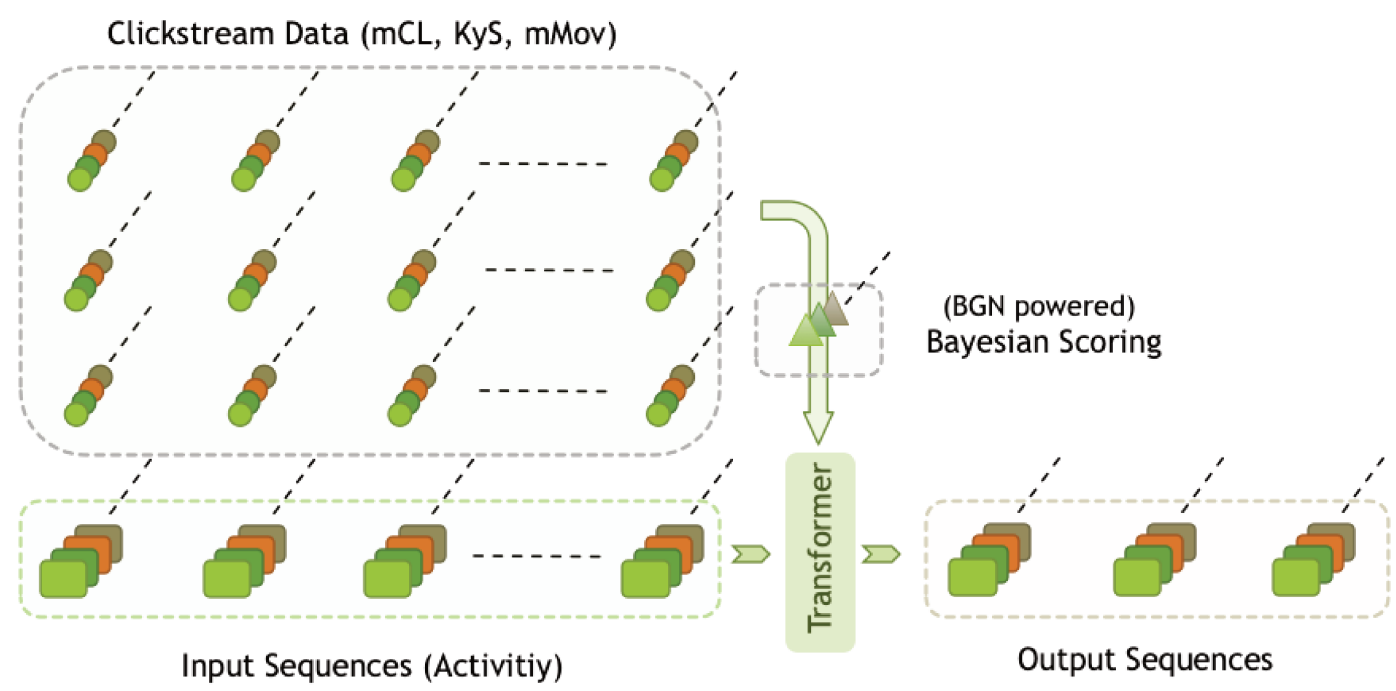

Figure 4.4: Clickstream Scoring Process

the following libraries: Pandas, Numpy, Sklearn, Math, Matplotlib, Tensorflow-backened Keras, and Statmodels.

\subsubsection{System implementation details}

This model consist in an Encoder-Decoder systems to perform the forecasting task in a Sequence-To-Sequence fashion. A representation of these Encoder-Decoder models is sketched in Figure 4.5. Three different approaches to the Sequence-To-Sequence model are built. The model breaks down the series of activities in chains of short subsequences that predicts upcoming next sub-secuences based on the clickstream recorded data. Each one of these model is based on a LSTM/GRU - RNN architecture. 


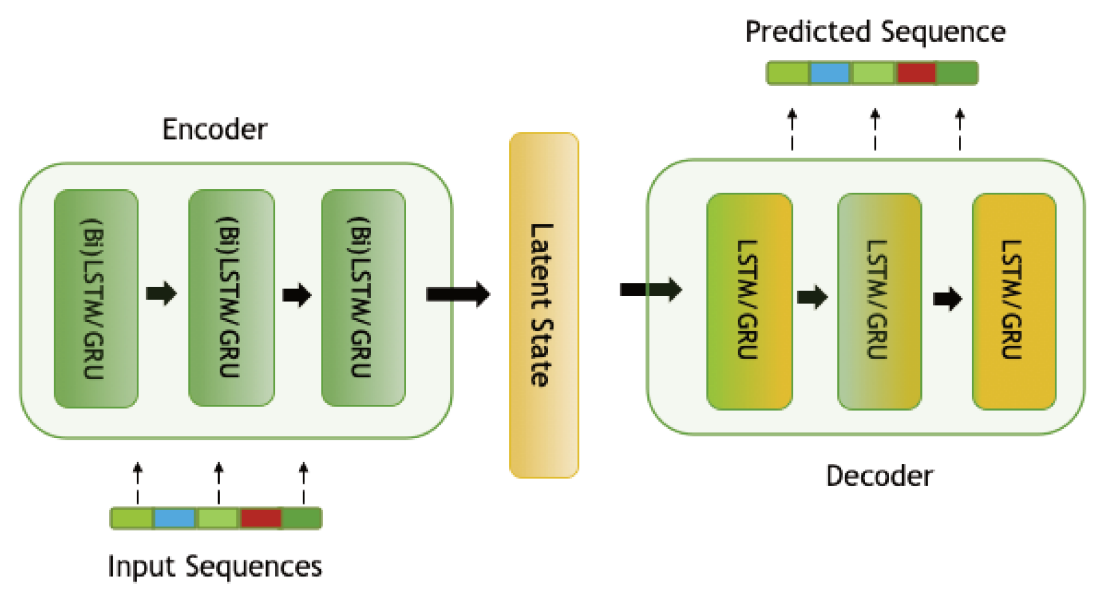

Figure 4.5: Sequence-to-Sequence Architecture

Four versions of the Encoder-Decoder architecture are presented:

- The first model: This consists in an Encoder-Decoder system where both, the encoder and the decoder features a stacked LSTM/GRU with two LSTM/GRU layers and Dropout layers as well.

- The second model: It is also an Encoder-decoder system where the encoder has up to two bidirectional composited LSTM/GRU layers and regular single layers in the decoder.

- Third model: Transformer model where the Encoder has two LSTM/GRU plus dropouts layers and the Decoder also has other two LSTM/GRU layers. The multiplicative attention (Loung Attention) layer is applied in the encoder-to-decoder transition.

- Fourth model: Transformer similar to he Third model, but now using Additive attention mechanism (Bahdanau Attention)

Now, the next model consists in an Hybrid Ensemble containing a BGN model coupled to a Transformer. The architecture details of the modified Transformer is shown in Figure 
4.6. The BGN is built using the clickstream data (i.e. keystrokes, left mouse clicks, and the duration in each activity by the user). A Bayesian score is computed with this BGN, which estimates the probability that the user chooses to go from activity $A$ to activity $B$, considering the current click data.

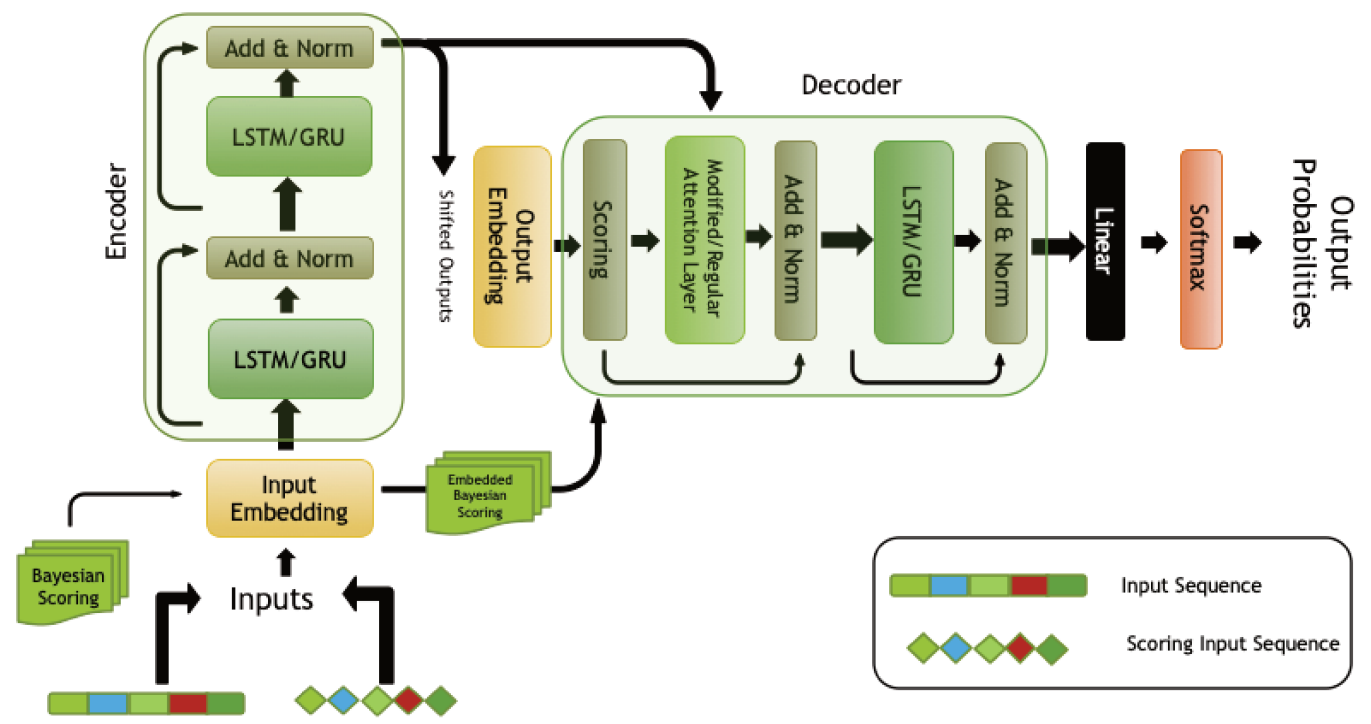

Figure 4.6: Modified Transformer Architecture

\section{Attention modification}

In this Hybrid Ensemble, the attention layer is modified by introducing a scoring factor which is computed through a Bayesian graph model. This Bayesian scoring factor will depend on every time-step in the clickstream data from the user during the particular activity he/she is at that time-step. The vector representation of each target step-value is multiplied by computed Bayesian score of its actual step value, this is

$$
v_{t}=v\left[A_{t}\right] \longrightarrow V_{t}^{B s c}=V^{B s c}\left[A_{t}\right]=v_{t} x S_{B G N}\left[A_{t}\right]
$$


When the data is entered in the Transformer architecture, for each input sequence its Bayesian-scored copy is also entered to the input layer of the RNN. The alignment-score to determine context is computed similar to the Luong mechanism:

$$
S_{a t t}=H_{e n c}[T] \cdot H_{d e c}[B s c]+H_{e n c}[B s c] \cdot H_{d e c}[T]
$$

where

- $S_{a t t}:$ multiplicative alignment score

- $H_{\text {enc/dec }}[T]:$ encoder/decoder hidden state from Target embedding

- $H_{\text {enc/dec }}[B s c]:$ encoder/decoder hidden state from Bayesian-scored Target embedding.

A linear layer computes the vector of contexts before the output layer is reached by the data. The modified attention mechanism is adjusted throughout the hyper-parameters alfa, beta, and delta introduced to compute the Bayesian score. In case of other than the "Dot" method is used, the expression for the alignment score will be modified accordantly (Luong, Pham, and Manning, 2015), (Du et al., 2017), (Zhou et al., 2018). Essentially, the modification to the attention mechanism proposed here consist in applying a multivariate treatment to a highly random variable. This treatment is based on a new correlated variable which accounts for the underlying Bayesian process driving the model.

\subsection{Evaluation}

The experiments described in the following section evaluate the performance of the predictor and compare the model results to a baseline model developed in previous work. 
The predictor is an Encoder-Decoder RNN-powered system. The performance of the BGN as a useful tool to modify the attention mechanism in Transformers is also tested. As described in the previous section, there are four model architectures to which experiments apply: 1) Model-1: feed-forward LSTM/GRU based sequence-to-sequence model, 2) Model-2: Bi(LSTM/GRU)-powered model, 3) Model-3: a Transformer model, featuring Loung attention mechanism; and 4) Model-4: another Transformer model with its attention mechanism modified by using a BGN.

\subsubsection{Complexity analysis}

One important challenge faced by this work is that the system has to be capable of processing incoming data continuously, while, at the same time, it should also continuously deliver opportune outputs in its role as a detection artifact. In order to achieve a competitive performance, the system should be able to perform multiple complex tasks in parallel, and this is addressed in the algorithm design. Another aspect considered in the design is the ability of self-adjusting hyper-parameters to better cope with time efficiency in the whole process of pattern detection. Considering that the predictor module (the BGN-Transformer) has to process a collection of extra features, the computational complexity will certaintly be increased, albeit only linearly with regards to the number of extra features.

\subsubsection{Predictive performance}

The performance of the predictor as presented in tables 2.1 to table 4.2 is measured in terms of accuracy, right-content, and accuracy in the first predicted state for the incoming subsequence. Average accuracy is defined as the feature of the predicted subsequence by which it fully matches the real subsequence. The right content shows the percentage of the real 
states from the real sequence that can be found in the predicted sequence. Accuracy in the first predicted state for the incoming sequence, as its name indicates, just measures the concordance of the first predicted state with the real first state in the incoming sequence.

TABLE 4.1.A: BGN Configuration for Transformer (GRU)

\begin{tabular}{llllllllllll}
\hline & \multicolumn{8}{c}{ BGN Clickstream configuration (mCL - KyS - mMov) } \\
\cline { 2 - 10 } BGN-Trasformer & $\begin{array}{l}\text { Baseline } \\
\text { (LSTM) }\end{array}$ & $\begin{array}{l}\text { Model-3 } \\
\text { (GRU) }\end{array}$ & $\mathbf{0 - 0 - 0}$ & $\mathbf{1 - 0 - 0}$ & $\mathbf{0 - 1 - 0}$ & $\mathbf{0 - 0 - 1}$ & $\mathbf{1 - 1 - 0}$ & $\mathbf{1 - 0 - 1}$ & $\mathbf{0 - 1 - 1}$ & $\mathbf{1 - 1 - 1}$ \\
\hline Accuracy & $31 \%$ & $40 \%$ & $43 \%$ & $34 \%$ & $\mathbf{4 4 \%}$ & $38 \%$ & $37 \%$ & $36 \%$ & $37 \%$ & $39 \%$ \\
Right Content & $\mathbf{4 9 \%}$ & $43 \%$ & $45 \%$ & $41 \%$ & $\mathbf{4 9 \%}$ & $42 \%$ & $40 \%$ & $41 \%$ & $43 \%$ & $42 \%$ \\
$1^{\text {st }}$ State Accuracy & $32 \%$ & $45 \%$ & $49 \%$ & $43 \%$ & $\mathbf{5 1 \%}$ & $42 \%$ & $39 \%$ & $38 \%$ & $35 \%$ & $43 \%$ \\
\hline
\end{tabular}

A first set of experiments attempts to determine the best configuration of the clickstream data for the composition of the BGN. In other words, in terms of equation (4.2), the task of the second set of experiments is to find which of the set of parameter coefficients could be selected. This refers to the Bayesian probabilities associated to the observed variables, such as left mouse-clicks (mCL), key strokes (KyS), and mouse movements (mMov). Each column on Table 4.1.a that is named with a 3-character combination of zeroes and ones, represents the three parameters, $\alpha-\beta-\delta$ in equation 4.2 , which capture the three observed variables (mCL-KyS-mMov).

In Table 4.1.a, Model-4, the modified Transformer model (GRU), is compared with the baseline model from previous work (stacked LSTM), and with the standard Transformer (GRU version). The configuration where only the key-stroke variable is considered, that is column '0-1-0', shows the best performance of all BGN clickstream configurations. When the '0-1-0' 
leading configuration is compared to the baseline model for accuracy and first-state accuracy, the improvement is significant, with more than 10 percentage points above baseline. For the right-content score, the leading configuration matches the baseline performance. Comparing the leading configuration to the standard Transformer model (Model-3) the experiments shows superior performance of the model developed in the previous chapter in all three comparative scores. The second best BGN clickstream configuration, that is '00-0', shows better performance for accuracy and first-state accuracy when compared with both baseline and Model-3. It should be noted from equation (4.2) that this configuration also benefits from the Markov-Chain transition probability of the BGN.

TABLE 4.1.B: BGN Configuration for Transformer (LSTM)

\begin{tabular}{lccccc}
\hline & \multicolumn{5}{c}{ BGN config. (mCL - KyS - mMov) } \\
\cline { 2 - 6 } Metrics & Baseline (LSTM) & Model-3 (LSTM) & $\mathbf{0 - 0 - 0}$ & $\mathbf{0 - 1 - 0}$ & $\mathbf{1 - 1 - 1}$ \\
\hline Accuracy & $31 \%$ & $34 \%$ & $36 \%$ & $\mathbf{3 7 \%}$ & $\mathbf{3 7 \%}$ \\
Right Content & $\mathbf{4 9 \%}$ & $36 \%$ & $39 \%$ & $41 \%$ & $42 \%$ \\
$1^{\text {st }}$ State Accuracy & $32 \%$ & $36 \%$ & $\mathbf{4 3 \%}$ & $39 \%$ & $42 \%$ \\
\hline
\end{tabular}

In Table 4.1.b, the analysis is reduced to three configurations. The selection for Table 4.1.b is as follows: no BGN (alpha-beta-delta) parameter selected (that is all zeroes), all BGN parameters (all ones), and the best performer from Table 1 ('0-1-0'). From Table 4.1.b, it is clear that the proposed model can offer better performance when compared to both baseline and Model-3 for accuracy and first-state accuracy, while the baseline model still behaves better for the right-content score. 
The four model architectures from Model-1 to Model-4 and experiments results are listed on Table 4.2. For Model-4, Table 4.2 shows results for the best configuration of BGN stream data that was found in the experiments.

TABLE 4.2: Predictive Capability of Model's Architectures

\begin{tabular}{lllllllll}
\hline \multirow{2}{*}{ Metrics } & \multicolumn{8}{c}{ Model Version } \\
\cline { 2 - 9 } & $\begin{array}{l}\text { Baseline Model-1 } \\
\text { (LSTM) }\end{array}$ & $\begin{array}{l}\text { Model-1 } \\
\text { (GRT) }\end{array}$ & $\begin{array}{l}\text { Model-2 } \\
\text { (LSTM) }\end{array}$ & $\begin{array}{l}\text { Model-3 } \\
\text { (LSTM) }\end{array}$ & $\begin{array}{l}\text { Model-3 } \\
\text { (GRU) }\end{array}$ & $\begin{array}{l}\text { Model-4 } \\
\text { (LSTM) }\end{array}$ & $\begin{array}{l}\text { Model-4 } \\
\text { (GRU) }\end{array}$ \\
\hline Accuracy & $31 \%$ & $26 \%$ & $33 \%$ & $18 \%$ & $34 \%$ & $40 \%$ & $37 \%$ & $\mathbf{4 4 \%}$ \\
Right Content & $\mathbf{4 9 \%}$ & $29 \%$ & $37 \%$ & $20 \%$ & $36 \%$ & $43 \%$ & $41 \%$ & $\mathbf{4 9 \%}$ \\
$1^{\text {st }}$ State Accuracy & $32 \%$ & $29 \%$ & $42 \%$ & $28 \%$ & $36 \%$ & $45 \%$ & $39 \%$ & $\mathbf{5 1 \%}$ \\
\hline
\end{tabular}

A third set of experiments shown in Table 4.2 wishes to test the accuracy and contents of model results. In terms of accuracy, overall GRU versions for all proposed models show better results than the baseline case (33\% matching reality). The GRU version of Model-4 especially shows that the inclusion of a BGN notably improves results (44\%). This result is also noted in the GRU version of Model-3 with a regular Transformer (40\%). Right-content performance in the baseline model, a stacked LSTM, is still competitive and the best runner is the only option that reaches the same standards (49\% of right contents to predict the states). Finally, in predicting the first state of the incoming subsequence the best performer, the GRU version of Model-4 outperforms all other approaches with a highest score of $51 \%$ accuracy, compared to a lower $32 \%$ in the baseline case, and above 6 percentage points over the regular Transformer (version GRU of Model-3 at 45\%). 


\subsubsection{Pattern detection performance}

Finally, the tradeoff between running time and performance will be discussed in more detail. The computer setting in which all experiments were carry out was MacBook Pro $(2.3 \mathrm{GHz}$ Intel Core i7 16GB). The chart in Figure 4.7 shows the tradeoff between the additional time required for the model to run and the performance gains from the upgrade applied to the predictor component of the smart classifier; built in Chapter 3.

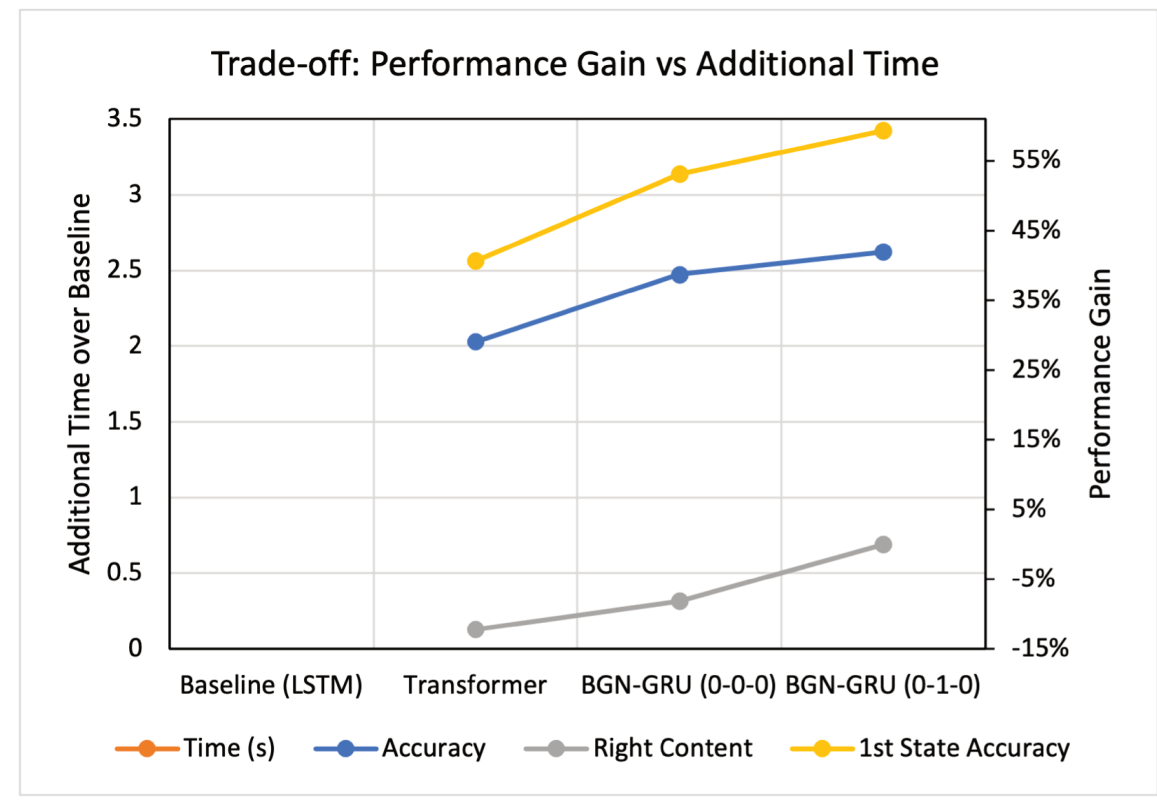

Figure 4.7: Tradeoff: Time vs Performance

\section{Efficiency analysis: Time vs Performance tradeoff}

The additional running time rate $Q_{\Delta T}$ was estimated with the use of:

$$
Q_{\Delta T}=\frac{\Delta T_{\text {Model }_{i}}-\Delta T_{\text {Baseline }}}{\Delta T_{\text {Baseline }}}
$$

and for the gain rates in Accuracy, Right Content, and 1st State Accuracy $Q_{\text {Score }}$ : 


$$
Q_{\text {Score }}=\frac{\text { Score }_{\text {Model }_{i}}-\text { Score }_{\text {Baseline }}}{\text { Score }_{\text {Baseline }}}
$$

When processing data from about one hundred students, in its baseline version the typical running time of each iteration of the Smart-Classifier is roughly 6 to 8 minutes. The graph shows that increasing the running time of each iteration from $\times 2$ to $\times 3.5$ leads to an improvement in performance reaching up to $42 \%$ and $59 \%$ over baseline scores for Accuracy and 1st State Accuracy, respectively.

In the classification task the system looks at subsequences of three to five consecutive activities, and according to the recorded data, a typical student spends around 10 minutes on average in each activity. Each iteration of the Smart Classifier has a running time of up to 0.3 minutes, when processing the data for each individual student. In that scenario, considering that the upgraded predictor increases the running time by 3.5 times; this represents roughly a 1.1-minute increase, which is a considerably small delay, compared to the average time per activity, which is about 10 minutes.

\subsection{Discussion}

In summary, the proposed Hybrid BGN-Transformer approach improves the overall performance of the Smart Classifier, while keeping running time under a reasonable range (Amezaga and Shafiq, 2021b). It is worth mentioning that the significative improvement achieved for the predictor module in particular, and for the overall system in terms of performance is in fact a promising open door for future improvements and refining in clickstream data processing in general. However, there are still some challenges to be addressed. 
Potential long-term correlations in the target variable are continuously overridden by the BGN scoring, which keeps no memory beyond a couple of time periods preceding the current stage. This limitation could be addressed by a different approach, for example, considering reinforcement learning instead of guidance through the BGN. In this case, the learning process would be more time consuming, and this would be difficult to accommodate in the limited time-window that individual LMS sessions typically offer. 


\section{Chapter 5}

\section{Conclusions and Future Directions}

This chapter summarizes the novelties in this work. This research shows that modelling sequential data generated in real-time by using Markov-Chain based approaches, combined with a Bayesian Graph Network coupled to an RNN, allows to detect patterns in the data in quasi-real time fashion. This solution could be pottentially used in the implementation of a tool to assist users interacting with an LMS or other monitoring applications.

Returning to the goals of this work and its contributions, we worked first with how to detect both regular and outlier patterns in clickstream data from an LMS, and how the appearance of outlier patterns in the data could be anticipated, so that the detection task could occur in quasi-real time. In this regards, we developed a hybrid model, the Smart Classifier, that is able to deliver a consistently good performance at all stages of its running cycle. In other words, a model which combine Markov-Chain based methods with a recurrent neural network, that is able to perform a reliable pattern-detection task over real-time clickstream data at every stage of an online working session.

As a second purpose and results, we focused on the popssibility of introducing a measurable 
score that could capture some hidden dynamics in the data-generation process that could be used to enhance the overall performance of the pattern-detection task. For this purpose, we used a Bayesian Graph Network as a tool for feature engineering to help modify the attention mechanism in the Transformer model. The resulting BGN-Transformer model enables the Smart Classifier to better capture underlying relationsips to improve its overall performance. With the data used in this work, this BGN-Transformer model predicts the next activity on a sequence of online activities that the user generates while interacting with an LMS. One of the main applications of this work is to improve the predictor module of a Smart Classifier.

This research also illustrates how some features in clickstream data can be modelled using a probabilistic approach to customize the attention mechanism in Transformer models. The results of this research could be potentially applied to the development of tools for assisting users in an online learning environment where timely assistance could be useful. The method also provides a systematic method to customize the attention mechanism in Transformer models that can be applied to a range of problems involving clickstream data.

\subsection{Conclusions}

The results achieved in the experiments of this work show a few key insights and guidelines for future work. The use of a hybrid system that combines a classic stochastic model with a neural network allows early detection of outliers in monitoring student online activity. Results show that the hybrid approach presented here (HMM-LSTM), using both One-Signal and Multi-Signal configuration, outperforms other approaches reviewed in the literature. 
The usability of the system is measured as a combination of how well the model behave as a classifier, and as pattern-detector when monitoringing clicstream data generated in quasi-real time. The proposed system delivers customized analysis in quasi-real time for each individual datagenerator.

The threshold selection is also an essential decision. Using a restrictive threshold increases the sensibility of the system, as presented in the results. In the context of students interacting with an LMS, a potential monitoring system based in this model with too low sensibility could lead to an inefficient management of resources in a lab session.

Performance results of the predictor component of the Smart Classifier introduced in Chapter 3, initially based on a standard LSTM architecture, suggest that performance could be improved by using a sequence-to-sequence approach. Transformer models have an attention mechanism which allows to capture relevant correlations in the data to improve the predictive task. With that in mind, as described in Chapter 4, the task of customizing such mechanism is successfully addressed by using a hybrid model combining a BGN and a Transformer model. This results in a better performance capacity in the forecasting task. This approach promises to be very useful to process data that is progressively generated. This is a common scenario when analyzing clickstream data from an LMS in quasi-real time.

Results discussed in Chapter 4 show that the BGN-Transformer approach followed in this research improves the predictor module of the solution that had been presented in Chapter 3. Enhanced results were obtained for the key metrics, accuracy, right-content, and firststate accuracy. The upgraded predictor component has the ability to provide a consistently 
better forecast for the first state of the next subsequence of activities, which better enables the system to produce a timely response. This is a key system feature that could be continued in future work. A key conclusion is that the use of a BGN to help the system understand underlying processes in the dynamic-data-generation that could substantially improve performance at the early running stages of an AI-powered monitoring system.

The possibility to include modifications to the way the alignment scores are computed and how relevant dependencies in the data are captured in Transformer models could be anticipated from the work of one of the Transformer pioneers (Luong, Pham, and Manning, 2015). With this work, however, a concrete example is explored, and this approach can be extended to other problems.

\subsection{Future work}

In future work, a reinforcement learning approach to assist the predictor component will be explored. The focus could be on the evaluation of the clickstream data as proxy to infer user intentions and its contribution to improving the entire detection system, that is the Smart Classifier. A possible improvement could be to individually optimize a neural network architecture for each session. This could significantly improve the predictive capacity of the system and, consequently, the overall performance of the detection task.

A key approach presented in this work is to modify the Transformer's attention mechanism. This could be applicable in future work to a wider range of problems in which there are extra features in the data that can provide insights about the underlying process that drives 
the evolution of the target variable.

The insights delivered by the solution could lead to valuable help for both students and professors interacting in quasi-real time within an LMS. Future work avenues could attempt to connect detected patterns from clickstream data from an individual LMS online session from one student with other data from the same student with the aim to predict behaviour and provide better assistance in future sessions.

Finally, exploring other combinations of deep-RNNs architectures coupled to Bayesian Graph Networks and other statistic methods, such as genetic algorithms, could help to better interpret the outcomes of hybrid deep-learning models. 


\section{Bibliography}

Amezaga, Alexis and M. Omair Shafiq (2021a). "Modeling and Predicting Online Activities of Students: An HMM-LSTM Hybrid Approach”. In: Manuscript submitted for publication.

- (2021b). “Transformer with Modified Attention Mechanism powered by a Bayesian Graph Network”. In: Manuscript submitted for publication.

Begleiter, Ron, Ran El-Yaniv, and Golan Yona (Dec. 2004). "On Prediction Using Variable Order Markov Models”. In: F. Artif. Int. Res. 22.1, pp. 385-421. ISSN: 1076-9757.

Borisov, Alexey et al. (Apr. 2016). “A Neural Click Model for Web Search”. In: Proceedings of the 25th International Conference on World Wide Web. International World Wide Web Conferences Steering Committee. DOI: 10 . 1145 / 2872427 . 2883033. URL: https://doi.org/10.1145/2872427.2883033.

Boukerche, Azzedine, Lining Zheng, and Omar Alfandi (July 2020). “Outlier Detection”. In: ACM Computing Surveys 53.3, pp. 1-37. DOI: 10 . 1145 / 3381028 . uRL: https : //doi.org/10.1145/3381028.

Cantabella, Magdalena et al. (Jan. 2019). “Analysis of student behavior in learning management systems through a Big Data framework”. In: Future Generation Computer Systems 90, pp. 262-272. DOI: 10 . $1016 /$ j . future . 2018.08 .003. uRL: https : //doi.org/10.1016/j.future.2018.08.003. 
Chang, Joshua C. (Mar. 2019). "Predictive Bayesian selection of multistep Markov chains, applied to the detection of the hot hand and other statistical dependencies in free throws". In: Royal Society Open Science 6.3, p. 182174. DOI: 10 . 1098/ rsos . 182174. URL: https://doi.org/10.1098/rsos. 182174.

Chatzigeorgakidis, Georgios et al. (2019). "Local Similarity Search on Geolocated Time Series Using Hybrid Indexing”. In: Proceedings of the 27th ACM SIGSPATIAL International Conference on Advances in Geographic Information Systems. SIGSPATIAL '19. Chicago, IL, USA: Association for Computing Machinery, pp. 179-188. ISBN: 9781450369091. DOI: 10 . 1145 / 3347146 . 3359349. urL: https : / / doi . org / 10 . 1145 / 3347146.3359349.

Chen, Chaochao et al. (Oct. 2018). "Semi-Supervised Learning Meets Factorization: Learning to Recommend with Chain Graph Model”. In: ACM Trans. Knowl. Discov. Data 12.6. ISSN: 1556-4681. DOI: 10 . 1145 /3264745. uRL: https : / / doi . org / 10 . 1145 / 3264745.

Cirstea, Razvan-Gabriel et al. (2018). "Correlated Time Series Forecasting Using Multi-Task Deep Neural Networks”. In: Proceedings of the 27th ACM International Conference on Information and Knowledge Management. CIKM '18. Torino, Italy: Association for Computing Machinery, pp. 1527-1530. ISBN: 9781450360142. DOI: 10 . $1145 / 3269206$. 3269310. uRL: https : / / doi.org/10 . 1145/3269206 . 3269310.

Deshpande, Prathamesh and Sunita Sarawagi (2019). "Streaming Adaptation of Deep Forecasting Models Using Adaptive Recurrent Units". In: Proceedings of the 25th ACM SIGKDD International Conference on Knowledge Discovery and Data Mining. KDD '19. Anchorage, AK, USA: Association for Computing Machinery, pp. 1560-1568. ISBN: 9781450362016. 
DOI: $10.1145 / 3292500.3330996$. uRL: https : / / doi .org/10 $1145 /$ 3292500.3330996.

Ding, Daizong et al. (2019). "Modeling Extreme Events in Time Series Prediction”. In: Proceedings of the 25th ACM SIGKDD International Conference on Knowledge Discovery \& Data Mining. KDD '19. Anchorage, AK, USA: Association for Computing Machinery, pp. 1114-1122. ISBN: 9781450362016. DOI: 10 . 1145 / 3292500 . 3330896. URL: https://doi.org/10.1145/3292500.3330896.

Du, Mengnan, Ninghao Liu, and Xia Hu (Dec. 2019). “Techniques for interpretable machine learning". In: Communications of the ACM 63.1, pp. 68-77. DoI: 10 . 1145/3359786. URL: https://doi.org/10.1145/3359786.

Du, Min et al. (2017). "DeepLog: Anomaly Detection and Diagnosis from System Logs through Deep Learning". In: Proceedings of the 2017 ACM SIGSAC Conference on Computer and Communications Security. CCS '17. Dallas, Texas, USA: Association for Computing Machinery, pp. 1285-1298. ISBN: 9781450349468. DOI: 10 . 1145/3133956 . 3134015. uRL: https://doi .org/10.1145/3133956.3134015.

Eichmann, Philipp et al. (2019). "Visual Exploration of Time Series Anomalies with MetroViz". In: Proceedings of the 2019 International Conference on Management of Data. SIGMOD '19. Amsterdam, Netherlands: Association for Computing Machinery, pp. 19011904. ISBN: 9781450356435 . DOI: 10 . 1145 / 3299869 . 3320247 . uRL: https : //doi.org/10.1145/3299869.3320247.

Fawaz, Hassan Ismail et al. (Mar. 2019). "Deep learning for time series classification: a review". In: Data Mining and Knowledge Discovery 33.4, pp. 917-963. Dor: 10 . 1007 / s10618-019-00619-1. uRL: https : / / doi .org/10 . 1007 /s10618019-00619-1. 
Filvà, Daniel Amo et al. (Apr. 2019). "Clickstream for learning analytics to assess students' behavior with Scratch". In: Future Generation Computer Systems 93, pp. 673-686. DOI: 10.1016/j . future . 2018 . 10 .057. urL: https : // doi.org/10 . $1016 /$ j.future.2018.10.057.

Gers, Felix A., Jürgen Schmidhuber, and Fred Cummins (Oct. 2000). "Learning to Forget: Continual Prediction with LSTM”. In: Neural Computation 12.10, pp. 2451-2471. DOI: 10 . 1162 / 089976600300015015 . uRL: https : //doi .org/10 . 1162 / 089976600300015015.

Graf, Sabine and Kinshuk (2008). "Analysing the Behaviour of Students in Learning Management Systems with Respect to Learning Styles”. In: Advances in Semantic Media Adaptation and Personalization. Springer Berlin Heidelberg, pp. 53-73. DOI: 10 . 1007 / 978-3-540-76361_3. uRL: https : //doi.org/10 . 1007/978-3-540$76361_{-} 3$.

Hammer, Hugo Lewi, Anis Yazidi, and B. John Oommen (Apr. 2018). "On the classification of dynamical data streams using novel “Anti-Bayesian” techniques”. In: Pattern Recognition 76, pp. 108-124. DOI: 10 . $1016 / \mathrm{j} \cdot$ patcog $\cdot 2017$. 10 . 031. uRL: https : //doi.org/10.1016/j.patcog.2017.10.031.

Hanif, Muhammad et al. (2017). "Hidden Markov Model for Time Series Prediction”. In: Journal of Asian Scientific Research 7.5, pp. 196-205. DoI: 10 . 18488/ journal . 2 . 2017 . 75 . 196 205. urL: https://doi .org/10 . 18488/journal . 2 . 2017.75 .196 .205$.

Hatt, Tobias and Stefan Feuerriegel (Apr. 2020). "Early Detection of User Exits from Clickstream Data: A Markov Modulated Marked Point Process Model”. In: Proceedings of The 
Web Conference 2020. ACM. DoI: 10 . 1145 / 3366423 . 3380238 . URL: https : //doi.org/10.1145/3366423.3380238.

Hendrycks, Dan, Mantas Mazeika, and Thomas Dietterich (Dec. 2018). "Deep Anomaly Detection with Outlier Exposure". In: arXiv e-prints, arXiv:1812.04606, arXiv:1812.04606. arXiv: 1812.04606 [cs. LG].

Huynh, Huy M. et al. (June 2020). "Efficient algorithms for mining clickstream patterns using pseudo-IDLists". In: Future Generation Computer Systems 107, pp. 18-30. Dor: 10 . 1016/j . future.2020.01.034. urL: https://doi.org/10.1016/j . future.2020.01.034.

Jamshed, Aatif, Bhawna Mallick, and Pramod Kumar (May 2020). "Deep learning-based sequential pattern mining for progressive database”. In: Soft Computing 24.22, pp. 1723317246. DOI: 10 . 1007 /s00500-020-05015-2. uRL: https : / / doi . org/ $10.1007 / \mathrm{s} 00500-020-05015-2$.

Jha, S., K. Tan, and R. A. Maxion (2001). "Markov Chains, Classifiers, and Intrusion Detection". In: Proceedings of the 14th IEEE Workshop on Computer Security Foundations. CSFW '01. USA: IEEE Computer Society, p. 206. DoI: 10 . 5555/872752 . 873519. URL: https://dl .acm.org/doi/10.5555/872752.873519.

Karadayı, Yıldız, Mehmet N. Aydin, and A. Selçuk Öğrenci (July 2020). “A Hybrid Deep Learning Framework for Unsupervised Anomaly Detection in Multivariate Spatio-Temporal Data". In: Applied Sciences 10.15, p. 5191. DoI: 10 . 3390/app10155191. uRL: ht tps : //doi.org/10.3390/app10155191.

Kumar, Lokesh et al. (2011). "A Hybrid Machine Learning System for Stock Market Forecasting". In: fournal of International Technology and Information Management 20.1. URL: https://scholarworks. lib.csusb.edu/jitim/vol20/iss1/3. 
Liu, Shuai, Guojie Song, and Wenhao Huang (Mar. 2020). "Real-time Transportation Prediction Correction using Reconstruction Error in Deep Learning”. In: ACM Transactions on Knowledge Discovery from Data 14.2, pp. 1-20. DOI: 10 . 1145 / 3369871. URL: https://doi.org/10.1145/3369871.

Luong, Thang, Hieu Pham, and Christopher D. Manning (Sept. 2015). "Effective Approaches to Attention-based Neural Machine Translation”. In: Proceedings of the 2015 Conference on Empirical Methods in Natural Language Processing. Lisbon, Portugal: Association for Computational Linguistics, pp. 1412-1421. Dor: 10 . 18653 /v1 /D15 - 1166. URL: https : / /www . aclweb . org/anthology/D15-1166.

Maratea, Antonio, Alfredo Petrosino, and Mario Manzo (Oct. 2017). "User Click Modeling on a Learning Management System”. In: International fournal of Human Capital and Information Technology Professionals 8.4, pp. 38-49. DoI: 10 . 4018 / i j hcitp . 2017100104 . uRL:https://doi.org/10.4018/ijhcitp. 2017100104.

Matsubara, Yasuko and Yasushi Sakurai (2019). “Dynamic Modeling and Forecasting of Time-Evolving Data Streams”. In: Proceedings of the 25th ACM SIGKDD International Conference on Knowledge Discovery and Data Mining. KDD '19. Anchorage, AK, USA: Association for Computing Machinery, pp. 458-468. ISBN: 9781450362016. DOI: 10 . $1145 / 3292500$.3330947. uRL: https : / / doi org/10 . 1145/3292500 . 3330947.

Mizera, Andrzej, Jun Pang, and Qixia Yuan (Sept. 2018). "Reviving the Two-State Markov Chain Approach”. In: IEEE/ACM Transactions on Computational Biology and Bioinformatics 15.5, pp. 1525-1537. DOI: 10 . 1109/tcbb. 2017 . 2704592 . uRL: https : //doi.org/10.1109/tcbb.2017.2704592. 
Özen, Serkan, Volkan Atalay, and Adnan Yazici (2019). “Comparison of Predictive Models for Forecasting Time-Series Data”. In: Proceedings of the 2019 3rd International Conference on Big Data Research. ICBDR 2019. Cergy-Pontoise, France: Association for Computing Machinery, pp. 172-176. ISBN: 9781450372015. DOI: 10 . 1145 / 3372454 . 3372482. uRL: https://doi.org/10 . 1145/3372454.3372482.

Schoot, Rens van de et al. (Jan. 2021). "Bayesian statistics and modelling”. In: Nature Reviews Methods Primers 1.1. DOI: 10 . 1038 / s43586-020-00001-2. URL: https : //doi.org/10.1038/s43586-020-00001-2.

Shadab, Shadman et al. (2020). "DeepDBP: Deep neural networks for identification of DNAbinding proteins”. In: Informatics in Medicine Unlocked 19, p. 100318. DOI: 10 . $1016 /$ j . imu . 2020 . 100318. urL: https : / / doi . org/10 . 1016/j . imu . 2020.100318.

Sutskever, Ilya, Oriol Vinyals, and Quoc V. Le (2014). "Sequence to Sequence Learning with Neural Networks”. In: Proceedings of the 27th International Conference on Neural Information Processing Systems - Volume 2. NIPS’14. Montreal, Canada: MIT Press, pp. 31043112.

Taha, Ayman and Ali S. Hadi (May 2019). “Anomaly Detection Methods for Categorical Data”. In: ACM Computing Surveys 52.2, pp. 1-35. DOI: 10 . 1145 / 3312739. URL: https://doi.org/10.1145/3312739.

Tian, Qiangxing et al. (2019). “Time Series Prediction with Interpretable Data Reconstruction”. In: Proceedings of the 28th ACM International Conference on Information and Knowledge Management. CIKM '19. Beijing, China: Association for Computing Machinery, pp. 2133-2136. ISBN: 9781450369763. DOI: 10 . 1145 / 3357384 . 3358141. URL: https : //doi.org/10.1145/3357384.3358141. 
Tran, Vu et al. (Oct. 2017). "Personalised Search Time Prediction using Markov Chains”. In: Proceedings of the ACM SIGIR International Conference on Theory of Information Retrieval. ACM. DOI: 10 . 1145 /3121050 . 3121085. uRL: https : / / doi . org / 10 . $1145 / 3121050.3121085$.

Vahdat, M. et al. (2015). "A learning analytics approach to correlate the academic achievements of students with interaction data from an educational simulator”. In: Design for Teaching and Learning in a Networked World. Springer International Publishing, pp. 352366. DOI: 10 . $1007 / 978-3-319-24258-3$. uRL: https : / / doi . org/10 . $1007 / 978-3-319-24258-3$.

Vaswani, Ashish et al. (2017). “Attention is All You Need”. In: NIPS'17. Long Beach, California, USA: Curran Associates Inc., pp. 6000-6010. ISBN: 9781510860964.

Wei, Honghao et al. (Dec. 2019). “QuickStop”. In: ACM SIGMETRICS Performance Evaluation Review 47.1, pp. 79-80. DOI: 10 . 1145 / 3376930 . 3376981 . URL: https : / / doi.org/10.1145/3376930.3376981.

Wu, Gene P. K. and Keith C. C. Chan (July 2020). "Discovery of Spatio-Temporal Patterns in Multivariate Spatial Time Series”. In: ACM/IMS Transactions on Data Science 1.2, pp. 122. DoI: 10 . 1145/3374748. uRL: https : //doi .org/10 . 1145/3374748. Wu, Liwei et al. (Sept. 2020). "SSE-PT: Sequential Recommendation Via Personalized Transformer”. In: Fourteenth ACM Conference on Recommender Systems. ACM. DOI: 10 . 1145 / 3383313 . 3412258. urL: https : / / doi . org / 10 . 1145 / 3383313 . 3412258.

Wu, Neo et al. (Jan. 2020). "Deep Transformer Models for Time Series Forecasting: The Influenza Prevalence Case”. In: arXiv e-prints, arXiv:2001.08317, arXiv:2001.08317. arXiv: 2001.08317 [cs. LG]. 
Yang, Zichao et al. (June 2016). "Hierarchical Attention Networks for Document Classification”. In: Proceedings of the 2016 Conference of the North American Chapter of the Association for Computational Linguistics: Human Language Technologies. San Diego, California: Association for Computational Linguistics, pp. 1480-1489. DoI: 10 . 18653/v1/ N16-1174. uRL: https : / /www . aclweb . org/anthology/N16-1174.

Yin, Jiaming et al. (2019). "Experimental Study of Multivariate Time Series Forecasting Models”. In: Proceedings of the 28th ACM International Conference on Information and Knowledge Management. CIKM '19. Beijing, China: Association for Computing Machinery, pp. 2833-2839. ISBN: 9781450369763. DOI: 10 . 1145 / 3357384 . 3357826 . URL: https : //doi.org/10.1145/3357384.3357826.

Zhong, Xiao and David Enke (June 2019). "Predicting the daily return direction of the stock market using hybrid machine learning algorithms”. In: Financial Innovation 5.1. DOI: 10 . 1186 /s40854-019-0138-0. uRL: https : //doi .org/10 . 1186 / s40854-019-0138-0.

Zhou, Yajun et al. (2018). “A Markov Chain Based Demand Prediction Model for Stations in Bike Sharing Systems". In: Mathematical Problems in Engineering 2018, pp. 1-8. DOI: 10 . 1155 / $2018 / 8028714$. urL: https : / / doi . org/10 . 1155/2018/ 8028714 . 